\title{
Understanding the combined effect of $k$-space undersampling and transient states excitation in MR Fingerprinting reconstructions
}

\author{
Christiaan C. Stolk and Alessandro Sbrizzi
}

\begin{abstract}
Magnetic resonance fingerprinting (MRF) is able to estimate multiple quantitative tissue parameters from a relatively short acquisition. The main characteristic of an MRF sequence is the simultaneous application of (a) transient states excitation and (b) highly undersampled k-space. Despite the promising empirical results obtained with MRF, no work has appeared that formally describes the combined impact of these two aspects on the reconstruction accuracy. In this paper, a mathematical model is derived that directly relates the time varying $R F$ excitation and the k-space sampling to the spatially dependent reconstruction errors. A subsequent in-depth analysis identifies the mechanisms by which MRF sequence properties affect accuracy, providing a formal explanation of several empirically observed or intuitively understood facts. New insights are obtained which show how this analytical framework could be used to improve the MRF protocol.
\end{abstract}

Index Terms - Error analysis, Experimental design, Magnetic Resonance Imaging, MR Fingerprinting, Quantitative MRI.

\section{INTRODUCTION}

Magnetic resonance fingerprinting (MRF) [1]-[3], aims at quantitatively reconstructing multiple tissue parameters from a relatively short sequence during which the magnetization is in the transient states. Imaging is performed between two excitation pulses by means of a strongly under-sampled readout scheme, for instance single-shot spirals or few radial spokes. As a consequence, the reconstructed snapshots exhibit strong Fourier aliasing artifacts which can be filtered-out by a subsequent matching filter step to recover the parameters of interest, typically the relaxation times $\left(T_{1}, T_{2}\right)$ and the proton density $(\rho)$. While different approaches have been proposed for the reconstruction of MRF data [4]-[9], they all rely on (I) transient state acquisitions, (II) some form of non-uniform Fourier transformation to the spatial domain [10], [11] and, with the exclusion of [9], (III) look-up table match.

While the empirical results of MRF are promising (as witnessed by the popularity of the method [12]-[14]) there is, at the time of writing, not much work dedicated to the

Manuscript submitted on October 5, 2018.

Christiaan C. Stolk is with the University of Amsterdam, Science Park 107, 1098 XG, Amsterdam, The Netherlands (e-mail: C.C.Stolk@uva.nl).

Alessandro Sbrizzi is with the University Medical Center, Utrecht, Heidelberglaan 100, 3584 CX, Utrecht, The Netherlands (e-mail: a.sbrizzi@umcutrecht.nl). analysis of the error in the reconstructions. In particular, there is no theory providing quantitative information on the errors originating from the interplay of transient states excitation and $k$-space undersampling. To tackle the difficulty of the problem, over-simplistic assumptions are usually made. For example, the Fourier aliasing artifacts are usually considered to be (a) independent on the parameter maps, (b) identically and independently distributed (i.i.d.) and (c) having zero mean. Some sensitivity studies have been performed on the basis of this assumption and standard statistical techniques such as the Cramer-Rao lower bound have been applied [9], [15], [16]. However, these simplifications are not a satisfactory choice for an in-depth analysis and understanding of MRF because: (1) undersampling artifacts are correlated with the parameter maps; (2) images reveal structure, which thus is reflected in the nature of the Fourier aliasing meaning that the underlying parameter maps are also structured; (3) the intensity of undersampling perturbations depends on the specific moment during the transient sequence or, in other words, the noise in the images is heteroscedastic; (4) the size of the Fourier aliasing artifacts and the extent to which they can be assumed to be zero-mean, i.i.d. and normally distributed depend on the experimental settings, hence for the sake of generalization it is better not to make these restrictive assumptions.

Clearly, a deeper understanding of the MRF error in relation to experimental design choices is urgent and important; this is the aim of our work. Leveraging on techniques from perturbation theory, we derive a mathematical model which explicitly relates the combined effect of RF excitation train and $k$-space under-sampling scheme to the systematic error in the reconstructed parameter maps. No assumptions are made with regard to the distribution of the noise terms which are instead treated for what they are, that is, Fourier aliasing perturbations.

Based on our model, we are able to identify the situations when MRF works, in the sense that the errors in the reconstructed parameters are sufficiently small to be reasonably ignored. We also describe the scenarios for which MRF fails and provide mathematical justifications for that. In addition, we address the role of randomness and structure in the $k$-space acquisition and RF excitation train obtaining results which are somehow unexpected from an intuitive point of view. We also indicate how the analytical techniques introduced in this work could be leveraged to further improve the MRF protocol.

The paper is organized as follows. Section [II introduces the 
basic concepts and describes the MRF signal model. Section III proceeds with the perturbation theoretic analysis of the error and identifies the terms which are responsible for the dominant biases. This is the main innovation of our work and culminates with equations (25) and 27). In Section IV] several tests are reported to validate the proposed model in realistic acquisition/reconstruction scenarios. Subsequently, in Section $\mathrm{V}$ we derive general insights into various aspects of MRF such as the role of randomness, cross-talk effects between parameters and transient RF excitation. Finally, a general discussion is provided in Section VI

\section{THE MRF FRAMEWORK}

\section{A. Signal model for under-sampled k-space acquisitions}

In this section we introduce a model for the MRF signal in the case of two-dimensional imaging. The acquisition sequence contains $N_{\mathrm{I}}$ radiofrequency (RF) pulses, with time $T_{R}$ between subsequent pulses and time-varying flip angles and phases given by $\alpha$ and $\phi$. By $\theta$ we denote a length $N_{\mathrm{P}}$ vector of local parameters which include the relaxation times $T_{1}, T_{2}$ and the proton density $\rho$. The discretized spatial domain is represented by a rectangular mesh $G_{\mathrm{p}}$ of size $m_{1} \times m_{2}$, with grid spacing 1 , defined by

$$
\begin{aligned}
G_{\mathrm{p}}=\left\{-\left\lfloor m_{1} / 2\right\rfloor, \ldots,-\left\lfloor m_{1} / 2\right\rfloor+m_{1}-1\right\} & \\
& \times\left\{-\left\lfloor m_{2} / 2\right\rfloor, \ldots,-\left\lfloor m_{2} / 2\right\rfloor+m_{2}-1\right\} .
\end{aligned}
$$

Position on the spatial grid will be denoted by $x=\left(x_{1}, x_{2}\right)$.

A first approximation to the signal from the macroscopic object, without thermal noise and spatial discretization effects, is then given by:

$$
\begin{aligned}
s_{j, l}=\sum_{x \in G_{\mathrm{p}}} M_{j}(\theta(x)) e^{-i k_{j, l} \cdot x}, & \\
& \text { with } j=1, \ldots, N_{\mathrm{I}} \text { and } l=1, \ldots, N_{\mathrm{RO}}
\end{aligned}
$$

where $M_{j}$ is the magnetization at the $j$-th echo, $N_{\mathrm{RO}}$ is the total number of read-outs (i.e. snapshots) and $k_{j, l}$ are the $k$-space sampling locations of the $l$-th sample during the $j$-th readout interval. Due to our choice of spatial grid, $k_{j, l} \in[-\pi, \pi]^{2}$. Note that $j$ denotes also the snapshot or frame index. Data is denoted by $d_{j, l}$ and has the same structure as the modeled signal but may contain thermal noise. The aim of our analysis is to investigate the interplay between the transient states spin evolution and the under-sampled $k$-space trajectory. Since we are interested in heavily under-sampled $k$-space acquisitions, we assume that the thermal error and the numerical approximation effects of the Discrete Fourier transform are negligible in comparison with aliasing artifacts and thus they will not be taken into account.

The $k$-space data is processed to a set of snapshot images $I$, defined by

$$
\begin{aligned}
I(x) & =\left(I_{1}(x), \ldots, I_{N_{\mathrm{I}}}(x)\right) \\
I_{j}(x) & =\frac{1}{m_{1} m_{2}} \sum_{l} w_{j, l} e^{i k_{j, l} \cdot x} d_{j, l}(k),
\end{aligned}
$$

where $x \in G_{\mathrm{p}}, j=1, \ldots, N_{\mathrm{I}}$ and $w_{j, l}$ are quadrature or $k$ space density compensation weights. By inserting Eq. (2) in Eq. (3) we obtain

$$
I_{j}(x)=\frac{1}{m_{1} m_{2}} \sum_{l} \sum_{y \in G_{\mathrm{p}}} w_{j, l} e^{i k_{j, l} \cdot(x-y)} M_{j}(\theta(y)) .
$$

Based on Eq. (4), we define the time-dependent point spread functions (PSF) associated with the $j$-th snapshot as

$$
P_{j}(x)=\frac{1}{m_{1} m_{2}} \sum_{l} w_{j, l} e^{i k_{j, l} \cdot x}
$$

thus Eq. (4) can be written as a convolution $I_{j}(x)=$ $\sum_{y \in G_{\mathrm{p}}} P_{j}(x-y) M_{j}(\theta(y))$. In the ideally Nyquist sampled $k$-space, each $P_{j}$ would resemble a delta function. In MRF this is not the case and $P_{j}$ causes strong aliasing artifacts in each snapshot image.

For a typical MRF sequence, the number of frames $N_{\mathrm{I}}$ is much larger than the undersampling factor and the $k$-space sampling is varied at each readout to achieve a full coverage of the spatial frequencies over the whole set of acquisitions. In other words, the average PSF defined as

$$
P(x)=\frac{1}{N_{\mathrm{I}}} \sum_{j=1}^{N_{\mathrm{I}}} P_{j}(x)
$$

describes a Kronecker delta for realistic MRF experiments and thus it can be considered an alias-free point spread function.

We define the undersampling errors by

$$
e_{\mathrm{US}, j}(x)=I_{j}(x)-P * M_{j}(\theta(\cdot))(x)
$$

where $*$ denotes convolution. Equivalently, we have:

$$
I_{j}(x)=P * M_{j}(\theta(x))+e_{\mathrm{US}, j}(x) .
$$

In MRF, for each $x$ the undersampling errors $e_{\mathrm{US}}(x)=$ $\left(e_{\mathrm{US}, j}(x)\right)_{j=1, \ldots, N_{\mathrm{I}}}$ are treated as i.i.d. normally distributed noise. This has implications in the sequence design. For example, in [1] it is argued that a certain amount of randomness in the choice of sequences (e.g. randomly varying $T_{\mathrm{R}}(j)$ and small random variations in $\alpha(j)$ ) should guarantee that this assumption is to a large degree satisfied. On the other hand in [9], [15], [16] it was argued that, taking into account statistical considerations for the least-squares estimator, optimal sequences are in fact highly structured, that is, flip angle values show clear temporal correlation. We should emphasize that in general the undersampling errors $e_{\mathrm{US}}(x)$ are not normally distributed and do not average out to zero, see also section 4 of the Supplementary material.

\section{B. Parameter reconstruction}

The MRF parameter reconstruction is defined as a leastsquares estimator $\theta^{*}(x)$ of $\theta(x)$ :

$$
\theta^{*}(x)=\underset{\theta}{\arg \min }\|I(x)-M(\theta)\|^{2} .
$$

To reduce the dimensionality of the minimization problem in Eq. (9), the fact that $M$ is linear in $\rho$ can be used. Let us denote $\theta=(\eta, \rho)$, in case $\rho$ is taken as a real parameter, and $\theta=(\eta, \operatorname{Re} \rho, \operatorname{Im} \rho)$ in case $\theta$ contains the complex 
parameter $\rho$. Using the well known relation between leastsquares estimation and the so called matched filter estimation [4], $\theta$ can also be obtained as follows:

$$
\eta^{*}(x)=\underset{\eta}{\arg \max } \frac{|\langle I(x), M(\theta)\rangle|}{\|I(x)\|\|M(\theta)\|},
$$

where $\langle\cdot, \cdot\rangle$ denotes the usual complex inner product that is antilinear in the second argument, and

$$
\rho^{*}(x)=\frac{\left\langle I(x), M\left(\eta^{*}(x), 1\right)\right\rangle}{\left\|M\left(\eta^{*}(x), 1\right)\right\|^{2}} .
$$

In the MR fingerprinting experiments described in the literature, the maximization as given in Eq. (10) is typically implemented by using a pre-computed dictionary.

\section{A MOdEL FOR THE RECONSTRUCTION ERROR}

To obtain a model for the error, we first derive the equations that characterize the reconstructed parameters $\theta^{*}$. Note that $\theta^{*}$ is the stationary point of the least-squares objective function from Eq. (9). The corresponding normal equations are nonlinear and are difficult, if not impossible, to solve analytically. Therefore, we will expand $\theta$ and $\theta^{*}$ as:

$$
\begin{aligned}
\theta(x) & =\theta_{0}+\theta_{1}(x), \\
\theta^{*}(x) & =\theta_{0}+\theta_{1}^{*}(x)
\end{aligned}
$$

where $\theta_{0}$ is a spatially constant value and $\theta_{1}, \theta_{1}^{*}$ are the contrast terms in, respectively, the true parameter and the reconstruction. Subsequently, we will linearize $M(\theta)$ and its derivative $\mathcal{D} M(\theta)$ around $\theta=\theta_{0}$. The result will be an equation for $\theta_{1}^{*}(x)$, which describes the errors in MR fingerprinting reconstructions at each spatial location $x$.

We will show that $\theta_{1}^{*}$ can be written in the form

$$
\theta_{1}^{*}(x)=P * \theta_{1}(x)+\epsilon_{1}(x)+\epsilon_{2}\left(x, \theta_{1}(\cdot)\right)+\text { h.o.t. }
$$

where $P$ is the point spread function defined in Eq. (6) and $\epsilon_{1}$ and $\epsilon_{2}$ are error terms, the latter of which depends on the function $\theta_{1}$. The abbreviation h.o.t. stands for higher order terms in $\theta_{1}$ and in the derivatives $\mathcal{D} M$. These terms will be discarded in the subsequent analysis of Eq. (13). Note that the term $P * \theta_{1}(x)$ depends purely on the $k$-space sampling scheme and not on the dynamic behavior of the magnetization (thus it is independent on the RF excitation train). Furthermore, the term $\epsilon_{1}$ is independent of $\theta_{1}$, thus this error will in general be present even in the absence of contrast (homogeneous object).

Crucially, the two error terms $\epsilon_{1}(x)$ and $\epsilon_{2}(x)$ depend on functions $S_{1 ; p}^{(1,0)}(x), S_{1 ; p, q}^{(2,0)}(x)$ and $S_{1 ; p, q}^{(1,1)}(x)$. These are convolution kernels which contain information about the time evolution of the magnetization and $P_{j}(x)$ and capture the combined effects of undersampling and transient state sequences.

The rest of this section is dedicated to the derivation of Eq. (13). To simplify the exposition, we will start with the case of constant proton density in $\theta_{0}$. Afterward, we will consider the general case of spatially varying $\rho_{0}$.

\section{A. Stationary points of the MRF objective functions}

As already mentioned in the previous paragraph, the MRF estimate $\theta^{*}(x)$ is a stationary point of the objective function in Eq. (9). Therefore, $\theta^{*}(x)$ must satisfy the equations

$$
0=\operatorname{Re}\left\langle M\left(\theta^{*}\right)-I(x), \frac{\partial M}{\partial \theta_{p}}\left(\theta^{*}\right)\right\rangle, \forall x, \forall p=1, \ldots, N_{\mathrm{P}}
$$

for $p=1, \ldots, N_{\mathrm{P}}$. From equations (4) and (5) it follows that

$$
\begin{aligned}
& 0=\operatorname{Re} \sum_{j=1}^{N_{\mathrm{I}}} \overline{\mathcal{D} M\left(\theta^{*}(x)\right)_{j ; p}} M\left(\theta^{*}(x)\right)_{j} \\
& -\operatorname{Re} \sum_{j=1}^{N_{\mathrm{I}}} \sum_{y \in G_{\mathrm{p}}} P_{j}(x-y) \overline{\mathcal{D} M\left(\theta^{*}(x)\right)_{j ; p}} M(\theta(y))_{j}
\end{aligned}
$$

where $\mathcal{D} M(\theta)$ denotes the jacobian matrix of $M, \mathcal{D} M(\theta)_{j ; p}$ are its components and the overscoring indicates complex conjugation.

\section{B. Expansion of the terms in Eq. 15.}

The next step is to replace $\theta(x)$ and $\theta^{*}(x)$ by $\theta_{0}+\theta_{1}(x)$ and $\theta_{0}+\theta_{1}^{*}(x)$ and expand Eq. 15) to first order in $\theta_{1}(x)$ and $\theta_{1}^{*}(x)$. Using first order Taylor expansions for $M(\theta)$ and $\mathcal{D} M(\theta)$ around $\theta_{0}$, we straightforwardly obtain

$$
\begin{aligned}
& \overline{\mathcal{D} M\left(\theta^{*}(x)\right)_{j ; p}} M(\theta(y))_{j}=\overline{\mathcal{D} M\left(\theta_{0}\right)_{j ; p}} M\left(\theta_{0}\right)_{j} \\
& \quad+\sum_{q=1}^{N_{P}} \overline{\mathcal{D}^{2} M\left(\theta_{0}\right)_{j ; p, q}} M\left(\theta_{0}\right)_{j} \theta_{1, q}^{*}(x) \\
& +\sum_{q=1}^{N_{P}} \overline{\mathcal{D} M\left(\theta_{0}\right)_{j ; p}} \mathcal{D} M\left(\theta_{0}\right)_{j ; q} \theta_{1, q}(y)+\text { h.o.t. }
\end{aligned}
$$

Defining

$$
\begin{aligned}
& S_{p, q}^{(1,1)}(x)=\sum_{j=1}^{N_{I}} P_{j}(x) \overline{\mathcal{D} M\left(\theta_{0}\right)_{j ; p}} \mathcal{D} M\left(\theta_{0}\right)_{j ; q} \\
& S_{p}^{(1,0)}(x)=\sum_{j=1}^{N_{I}} P_{j}(x) \overline{\mathcal{D} M\left(\theta_{0}\right)_{j ; p}} M\left(\theta_{0}\right)_{j} \\
& S_{p, q}^{(2,0)}(x)=\sum_{j=1}^{N_{I}} P_{j}(x) \overline{\mathcal{D}^{2} M\left(\theta_{0}\right)_{j ; p, q}} M\left(\theta_{0}\right)_{j}
\end{aligned}
$$

we observe that, to first order, the second term in Eq. 15 may be written as

$-\operatorname{Re}\left[S_{p}^{(1,0)} * 1(x)+\sum_{q=1}^{N_{P}} S_{p, q}^{(1,1)} * \theta_{1, q}(x)+\sum_{q=1}^{N_{P}} \theta_{1, q}^{*} S_{p, q}^{(2,0)} * 1(x)\right]$

where $1(x)$ denotes the constant function with value 1 at all locations $x$ in the mesh.

The sums defined in Eqs. (17) are an essential element of the analysis. They are weighted sums of the (time-dependent) point spread functions, with the "weights" given by linearantilinear terms $\overline{\mathcal{D} M\left(\theta_{0}\right)_{j ; p}} M\left(\theta_{0}\right)_{j}$ etc. The sums contain the combined effects of $k$-space undersampling and timedependent behavior of the magnetization. 
We further split the terms in Eq. (15) into "mean" and "residual" parts. We therefore define

$$
\begin{aligned}
S_{\text {mean; }, q}^{(1,1)}(x) & =P(x) \sum_{j=1}^{N_{I}} \overline{\mathcal{D} M\left(\theta_{0}\right)_{j ; p}} \mathcal{D} M\left(\theta_{0}\right)_{j ; q} \\
S_{\text {resid } ; p, q}^{(1,1)}(x) & =\sum_{j=1}^{N_{I}}\left(P_{j}(x)-P(x)\right) \overline{\mathcal{D} M\left(\theta_{0}\right)_{j ; p}} \mathcal{D} M\left(\theta_{0}\right)_{j ; q}
\end{aligned}
$$

such that

$$
S_{p, q}^{(1,1)}(x)=S_{\text {mean; }, q}^{(1,1)}(x)+S_{\text {resid; } p, q}^{(1,1)}(x) .
$$

This decomposition separates the effects of time-varying (residual) and constant (mean) sampling. It will turn out that the error terms are proportional to the residual parts. Consistently with this, the residual component vanishes when there is no undersampling (i.e. $P_{j}=P$ ) and/or when the magnetization is in the steady states, which results into the "weights" $\overline{\mathcal{D} M\left(\theta_{0}\right)_{j ; p}} \mathcal{D} M\left(\theta_{0}\right)_{j ; q}$ being time-independent. This is the case for conventional MRI acquisitions.

Similarly, we define $S_{\text {mean; } p}^{(1,0)}(x), S_{\text {resid; } p}^{(1,0)}(x)$ and $S_{\text {mean; }, q}^{(2,0)}(x)$, $S_{\text {resid; } p, q}^{(2,0)}(x)$ replacing the weights in Eq. 19 by the weights used in defining $S_{p}^{(1,0)}(x)$ and $S_{p, q}^{(2,0)}(x)$, respectively.

We proceed with the first term in Eq. (15). Using again the Taylor expansions of $M(\theta)$ and $\mathcal{D} M(\theta)$ and the definition of mean and residual components, this term can be written as

$$
\begin{aligned}
\operatorname{Re}\left[S_{\text {mean } ; p}^{(1,0)} * 1(x)+\sum_{q=1}^{N_{P}} \theta_{1, q}^{*} S_{\text {mean } ; p, q}^{(1,1)} * 1(x)\right. & \\
& \left.+\sum_{q=1}^{N_{P}} \theta_{1, q}^{*} S_{\text {mean } ; p, q}^{(2,0)} * 1(x)\right] .
\end{aligned}
$$

Finally, in our expansion of Eq. (15), Eq. (20) is used (and the similar property for $S_{p}^{(1,0)}(x)$ and $S_{p, q}^{(2, \overline{0})}(x)$ ) to obtain some cancellations, and make a clear identification of error terms possible. The first order expansion of Eq. [15] is thus:

$$
\begin{aligned}
& 0=\operatorname{Re}\left[\sum_{q=1}^{N_{P}} \theta_{1, q}^{*} S_{\text {mean } ; p, q}^{(1,1)} *\right.-\sum_{q=1}^{N_{P}} S_{\text {mean } ; p, q}^{(1,1)} * \theta_{1, q} \\
&-S_{\text {resid } ; p}^{(1,0)} * 1-\sum_{q=1}^{N_{P}} S_{\text {resid } ; p, q}^{(1,1)} * \theta_{1, q} \\
&\left.-\sum_{q=1}^{N_{P}} \theta_{1, q}^{*} S_{\text {resid } ; p, q}^{(2,0)} * 1\right] .
\end{aligned}
$$

This was obtained as the terms $\pm S_{\text {mean } ; p}^{(1,0)} * 1(x)$ and $\pm S_{\text {mean }}^{(2,0)} *$ $1(x)$ cancel each other. The above equation is a formal expansion of Eq. 15 in the variables $\theta_{1}, \theta_{1}^{*}, S_{\text {resid;p }}^{(1,0)}, S_{\text {resid;p,q }}^{(2,0)}$ and $S_{\text {resid; } p, q}^{(1,1)}$.

\section{Error model for constant proton density reference}

Equation 22, is a linear system for the MRF estimate $\theta_{1}^{*}(x)$ for each $x$. As a next step, we write down the solution of this system and identify the correct contribution and the systematic errors as outlined in Eq. (13).
We first observe that the function $S_{\text {mean; } p, q}^{(1,1)}(x)$ is simply given by $P(x) N_{p, q}$ where $N_{p, q}$ is defined as

$$
N_{p, q}=\sum_{j=1}^{N_{\mathrm{I}}} \overline{\mathcal{D} M\left(\theta_{0}\right)_{j ; p}} \mathcal{D} M\left(\theta_{0}\right)_{j ; q}
$$

The PSF $P$ is well-behaved, that is, it approaches a Kronecker delta, thus $P * 1 \approx 1$. As a consequence, the first and second term in 22 can be approximated by, respectively, $\sum_{q} \operatorname{Re} N_{p, q} \theta_{1, q}^{*}(x)$ and $\sum_{q} \operatorname{Re} N_{p, q}\left(P * \theta_{1, q}(x)\right)$.

The fifth term in 22 is a product of two factors assumed to be small, namely of $\theta_{1}^{*}$ and of $S_{\text {resid; } p, q}^{(2,0)}$. Staying with our philosophy of keeping only the first order terms, we will omit it. Defining two vector valued functions

$$
\begin{aligned}
& E_{1 ; p}(x)=\operatorname{Re} S_{\text {resid;p }}^{(1,0)} * 1(x) \\
& E_{2 ; p}(x)=\operatorname{Re} \sum_{q} S_{\text {resid; }, q}^{(1,1)} * \theta_{1, q}(x),
\end{aligned}
$$

we conclude that $\theta_{1}^{*}(x)$ is given to first order approximation by

$$
\theta_{1}^{*}(x)=P * \theta_{1}(x)+(\operatorname{Re} N)^{-1}\left(E_{1}(x)+E_{2}(x)\right),
$$

where it was used that $P$ is real. The first term on the right hand side is identified as the correct image. The other two terms in Eq. 13 are given by $\epsilon_{j}=(\operatorname{Re} N)^{-1} E_{j}, j=1,2$.

\section{Error model for variable proton density reference}

So far, we have assumed that all components of $\theta$ are close to some constant reference value. In Section 1 of the Supplementary material a more refined model is derived that allows for a variable proton density. We briefly explain why this is of interest. Firstly, the proton density is always zero outside the object (air) and therefore can hence hardly be considered "nearly constant". The second reason is better illustrated in the $k$-space domain. Note that by taking the Fourier transform on both sides of the error $E_{1}$ defined in Eq. 24 we obtain:

$$
\widehat{E}_{1 ; p}(k)=\mathcal{F}\left\{\operatorname{Re} S_{\text {resid;p }}^{(1,0)} * 1\right\}(k) .
$$

where $\widehat{E}_{1 ; p}$ denotes the Fourier transform of $E_{1 ; p}$. Convolution by the constant function 1 becomes a product in the $k$-space with a Dirac delta centered at $k=0$, thus the previous expression vanishes for all $k \neq 0$. For $k=0$ we obtain:

$$
\widehat{S}_{\text {resid } ; p}^{(1,0)}(0)=\sum_{j=1}^{N_{\mathrm{I}}}\left(\widehat{P}_{j}(0)-\widehat{P}(0)\right) \overline{\mathcal{D} M\left(\theta_{0}\right)_{j ; p}} M\left(\theta_{0}\right)_{j}
$$

where $\widehat{P}_{j}(k)$ and $\widehat{S}_{\text {resid; } p}^{(1,0)}(k)$ denote the Fourier transforms of $P_{j}(x)$ and $S_{\text {resid; } p}^{(1,0)}$, respectively.

In case of radial or spiral sampling, the $k=0$ Fourier component is sampled at each interval, so that $\widehat{P}_{j}(0)$ is independent of $j$ and equal to $\widehat{P}(0)$. Therefore, for radial or spiral sampling, $E_{1 ; p}(x)$ effectively vanishes. By allowing for a variable proton density a better approximation for this type of error is found that does not vanish. 
In case of variable reference proton density the equivalent of Eq. 25 is given by (see the Supplementary material)

$$
\begin{aligned}
& \theta_{1}^{*}(x)=\left|\rho_{0}^{*}(x)\right|^{-2}(\operatorname{Re} N)^{-1} \times \\
& \left(\operatorname{Re} \overline{\rho_{0}^{*}(x)} N P *\left(\rho_{0} \theta_{1}\right)(x)+E_{1}(x)+E_{2}(x)\right),
\end{aligned}
$$

where $\rho_{0}^{*}(x)=P * \rho_{0}(x)$ and

$$
\begin{aligned}
& E_{1 ; p}(x)=\operatorname{Re} \overline{\rho_{0}^{*}(x)} S_{\text {resid;p}}^{(1,0)} * \rho_{0}(x) \\
& E_{2 ; p}(x)=\operatorname{Re} \overline{\rho_{0}^{*}(x)} \sum_{q} S_{\text {resid } ; p, q}^{(1,1)} *\left(\rho_{0} \theta_{1, q}\right)(x) .
\end{aligned}
$$

In regions where $\rho_{0}$ varies, $\theta_{1}^{*}$ is in general no longer equal to $P * \theta_{1}$. However, inside the object we typically choose $\rho_{0}$ constant, so that $\rho_{0}^{*}$ equals $\rho_{0}$ and the first term reduces again to $P * \theta_{1}$, which we assume is small. In other words, $\rho_{0}$ is a binary valued function (i.e. a mask) which attains 0 in the locations outside the brain (air)). Note that, in this case, the error terms of the generalized error model of Eq. (13) are given by $\epsilon_{j}(x)=\left|\rho_{0}^{*}(x)\right|^{-2}(\operatorname{Re} N)^{-1} E_{j}(x)$ and they satisfy the properties introduced just after Eq. (13).

\section{MODEL VALIDATION}

In this section, we will investigate the validity of Eq. 27) as an MRF error model by means of numerical examples. In particular, we will consider standard, well-established MRF acquisition schemes and we will show that the error predicted by Eq. 27) is indeed a good approximation of the error obtained by actual MRF reconstructions. Subsequently, in section $\mathrm{V}$ we will leverage on our model to investigate and uncover different aspects of the MRF paradigm.

The examples that follow focus on 2D gradient spoiled sequences with radial, spiral and Cartesian $k$-space samplings where $\theta=\left(\log T_{1}, \log T_{2}, \rho\right)$. The logarithmic change of variable is meant to scale the relaxation times to a similar range. Other quantities that may be important are, for example, the relaxation time $T_{2}^{*}$ in presence of intravoxel dephasing and the transmit source field $B_{1}^{+}$. To keep our analysis within practical constraints, we do not consider them.

\section{A. General simulation setup}

To model the spoiling gradient effects, we compute the macroscopic voxel signal as a sum of differently resonating isochromat responses, each of which is modeled using the Bloch equations. Additional phase accrual effects caused by off-resonance are not taken into account since we focus on gradient spoiled sequences. Detailed information regarding the signal simulations and image reconstructions is reported in Section 2 of the Supplementary material.

As it is common in MRF, an inversion pulse precedes a time dependent flip angle train. The flip angles vary between 0 and $60^{\circ}$ and have a $90^{\circ}$ phase with respect to the inversion pulse, see also Fig. 1. Although our analysis holds for any choice of echo and repetition times $\left(T_{\mathrm{E}}\right.$ and $\left.T_{\mathrm{R}}\right)$, including temporally varying values, in this work we consider only fixed (time independent) values: $T_{\mathrm{R}}=15 \mathrm{~ms}$ and $T_{\mathrm{E}}=7.5 \mathrm{~ms}$. This sequence will be referred to as sequence 1 . In this section three sampling schemes are employed: radial golden angle, spiral golden angle and Cartesian. The resolution is $128 \times 128$ voxels and the undersampling factors for each image are 32, 32 and 16, respectively. The undersampling factors are defined in the angular, radial and phase encoding direction, respectively. We opt for a milder undersampling factor in the Cartesian acquisition since, as we will show, this kind of sequences is more susceptible to undersampling artifacts; a factor of 32 would lead to impracticable results.

MR fingerprinting reconstructions are performed by solving Eq. (10) using a precomputed dictionary of complex signal evolutions $M\left(T_{1}, T_{2}, 1\right)$. Here $T_{1}, T_{2}$ are chosen in logarithmic mesh with grid distances approximately $0.5 \%$ for $T_{1}$ and approximately $1 \%$ for $T_{2}$. With double precision computations this lead to a dictionary of a manageable size of about $1 \mathrm{~GB}$, and to an accuracy that is sufficient to compare modeled and MRF errors in the subsequent analysis.

Error predictions according to our model are obtained by numerical solution of Eq. 27). The first term in Eq. 27) is defined to be the correct solution. Having specified the function $\rho_{0}(x)$, it is straightforward to compute all the quantities in 28, and to solve the matrix equation for $\theta_{1}^{*}(x)$ for all $x$ in the FOV. The computations are implemented in the Julia programming language [17]. The convolutions make use of NUFFT while the derivatives involved in the $S^{(\alpha, \beta)}$ are evaluated by automatic differentiation. In the numerical solution of 27,28, the most computationally intensive steps are the convolutions involving spatially dependent quantities. These are done by applying forward and adjoint NUFFTs for each index $j$. The remaining steps are relatively cheap. The whole process takes about 3 minutes on a 16 cpu linux machine.

\section{B. Test 1.1: checkerboard phantom}

As a first test, a checkerboard model with variations of $\pm 25 \%$ in $T_{1}$ and smaller variations in $T_{2}$ is considered. The parameter values $\left(T_{1}, T_{2}\right)=(750,70) \mathrm{ms}$ and $\left(T_{1}, T_{2}\right)=$ $(1250,90) \mathrm{ms}$ roughly correspond to typical white and gray matter values, respectively. The reference proton density, $\rho_{0}$, is chosen to be equal to the true proton density that is, 1 inside the checkerboard and 0 outside.

Figure 2 shows the validation results for this phantom and the three sampling schemes. Root mean squared (RMS) averages of the actual MRF errors, the predicted errors and the partial error contributions $\epsilon_{1}(x)$ and $\epsilon_{2}(x)$ are given in Table I Further examples of checkerboard phantoms for larger parameter variations are given in section 3 of the Supplementary material.

\section{Test 1.2: numerical brain model}

The second example concerns a numerical brain phantom [18]. In this case $\rho_{0}$ is chosen equal to 0.8 a.u. inside the head and zero otherwise. The acquisition and reconstruction setups are the same as in the previous test. The results are displayed in Figure 3 and summarized in Table 1 .

From these two validation tests we observe the following. (a) When parameters vary moderately (e.g. $\pm 25 \%$ compared to the reference value, or a contrast of about a factor 1.5) inside the FOV, the predicted imaging errors according to our 

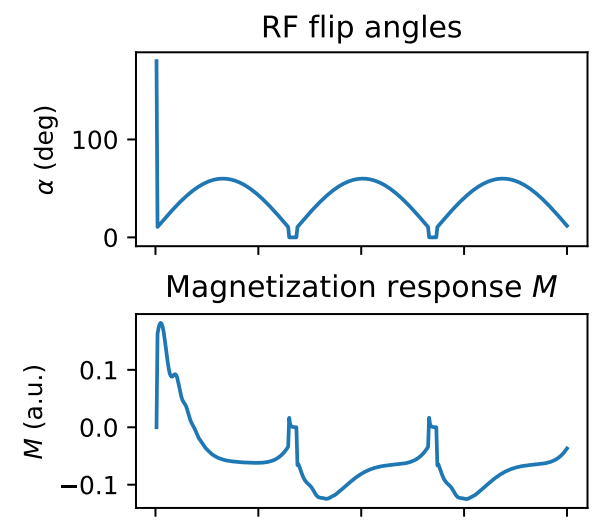

Derivatives of $M$

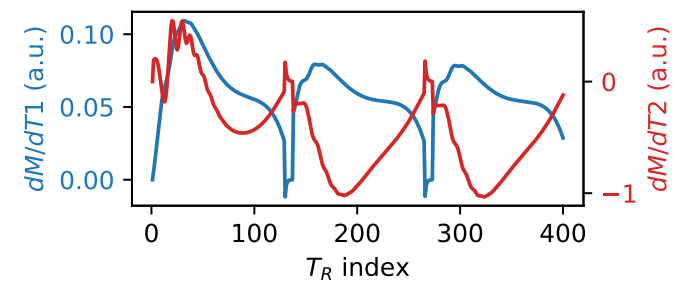

Fig. 1. Flip angles and plot of $M(t, \theta)$ and some derivatives for $\boldsymbol{T}_{1}=$ $1.0 \mathrm{~s}, T_{2}=0.08 \mathrm{~s}, \mathrm{PD}=1$ [a.u.], for sequence 1 .

model from Eq. 27) are in close agreement with the observed MRF imaging errors.

(b) When much larger parameter variations are present, some degradations occur, particularly in regions of extremely small or large parameters. Nonetheless, the overall error estimation is still qualitatively similar, and predicted and actually obtained errors are of the same order of magnitude. Therefore, Eq. 277) is still valid as a predictive error model.

(c) The Cartesian sampling is clearly a sub-optimal acquisition scheme and incapable of returning acceptable parameter maps in this 16-fold acceleration case.

\section{IN-DEPTH ANALYSIS}

To better understand how the choice of acquisition parameters affects the reconstructions, we will perform a more detailed analysis of the error terms $\epsilon_{1}$ and $\epsilon_{2}$ as modeled by Eqs. (25) and (27). We will take a Fourier domain perspective to directly connect the predicted errors with the data acquisition process. Note that, in the Fourier domain, the convolutions present in equations (24) and (28) become multiplications.

\section{A. The contrast independent error term $\epsilon_{1}$ and the role of variable density sampling}

We are going to show that the contrast independent error term $\epsilon_{1}$ is closely related to the sampling density around the center of the $k$-space. This error term is best modeled using variable $\rho_{0}$ as in (27) and is then given by $\left|\rho_{0}^{*}(x)\right|^{-2}(\operatorname{Re} N)^{-1} E_{1}(x)$ with $E_{1}$ as defined in 28). In the Fourier domain, the term $S_{\text {resid; } p}^{(1,0)} * \rho_{0}(x)$ becomes a multiplication between $\widehat{S}_{\text {resid }}^{(1,0)}(k)$ and $\widehat{\rho}_{0}(k)$. First of all, note that Eqs. (19) and (34) imply that frequent sampling at certain values of $k$ leads to smaller values of the coefficients $\widehat{S}_{\text {resid }}^{(1,0)}(k)$ at such $k$, cf. section $\mathrm{V}-\mathrm{C}$ Furthermore, $\left|\widehat{\rho}_{0}(k)\right|$ typically attains its largest values around $k=0$. Therefore, to minimize $\epsilon_{1}$ the weights $\widehat{S}_{\text {resid }}^{(1,0)}(k)$ should be small at $k \approx 0$, which is equivalent to frequent sampling at the center of $k$-space. Since $\left|\widehat{\rho}_{0}(k)\right|$ decays as $\sim 1 /|k|$ for large $|k|$ (a standard result from convergence of Fourier series for piecewise continuous functions), larger values of $\widehat{S}_{\text {resid }}^{(1,0)}(k)$ for large $k$ are allowed, leading to sparser sampling in the outer $k$-space region. In conclusion, employing a scheme which frequently samples the center of $k$-space leads to a small contrast-independent error contribution. With radial and spiral sampling, this naturally occurs. On the other hand, in regular Cartesian undersampling the point $k=0$ is sampled once every $m_{2} / N_{\mathrm{US}}$ times just like other values of $k$; in this case, larger values for the contrast independent error can be expected. This explains the large errors for Cartesian acquisition observed in section IV.

\section{$B$. The contrast dependent error $\epsilon_{2}(\mathrm{x})$ and parameters cross-talk}

The error term $\epsilon_{2}(x)$ depends linearly on the contrast $\theta_{1}(x)$, being the linear term in a Taylor expansion. This means that errors in the reconstructed $T_{1}$ and $T_{2}$ depend on the true $T_{1}$ and $T_{2}$ value maps and can thus inherit size and structure from them. In particular, one can expect cross-talk effects, which take place when the true value of $T_{1}$ influences the reconstruction of $T_{2}$ and vice versa. Here we will study in detail this phenomenon. To this aim, we consider the errors $\epsilon_{2}$, according to Eq. 25, which are given by $\epsilon_{2}(x)=(\operatorname{Re} N)^{-1} E_{2}(x)$ with $E_{2}(x)$ as given in Eq. (24). In the Fourier domain, there is thus a simple linear relation between the errors $\widehat{\epsilon}_{2}$ and the true contrast $\widehat{\theta}_{1}$ :

$$
\widehat{\epsilon}_{2}(k)=\mathcal{E}_{2}(k) \widehat{\theta}_{1}(k),
$$

where the $N_{\mathrm{P}} \times N_{\mathrm{P}}$ matrix $\mathcal{E}_{2}(k)$ is given by

$$
\mathcal{E}_{2}(k)_{p, q}=\sum_{r}(\operatorname{Re} N)_{p, r}^{-1} \widehat{S}_{\mathrm{resid} ; r, q}^{(1,1)}(k) .
$$

The off-diagonal coefficients of $\mathcal{E}_{2}(k)$ are direct indicators of cross-talk errors and only depend on the RF pulse sequence and $k$-space sampling scheme, not on the object being scanned. Therefore we will display some values of the $2 \times 2$ matrix block corresponding to the parameters $\log T_{1}$ and $\log T_{2}$. We will initially consider radial golden-angle $k$-space sampling.

As a first illustration, consider the checkerboard phantom and the images in Fig. 2. This phantom is characterized by large $k$-space components (not shown) at $k=$ $( \pm 0.08 \pi, \pm 0.08 \pi)$. For these values of $k$, the matrix $\mathcal{E}_{2}(k)$ is reported in Table II] at different undersampling rates. First of all, note that as the undersampling factor increases, also the entries of $\mathcal{E}_{2}(k)$ increase (in absolute value). This is supported by the basic intuition that the larger the undersampling, the larger the artifacts will be. Furthermore, the large $(2,1)$ component of $\mathcal{E}_{2}(k)$ for $N_{\mathrm{US}}=32$ show that the $T_{2}$ reconstruction is likely to receive a strong imprint from the true $T_{1}$ contrast. Indeed, in Figure 2 (second row, error columns) the overestimation of the $T_{2}$ contrast is clearly visible in the form of a $2 \mathrm{D}$ sinusoidal pattern. 
(a) radial
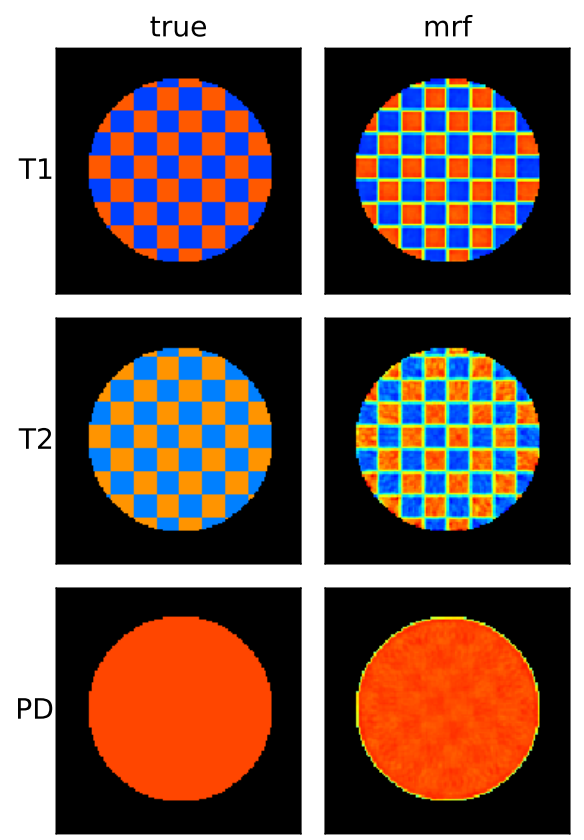

(c) spiral
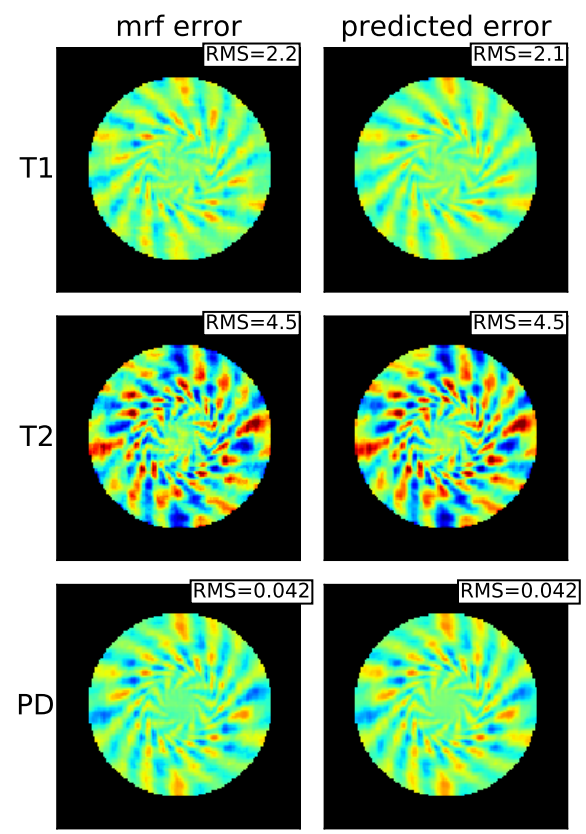
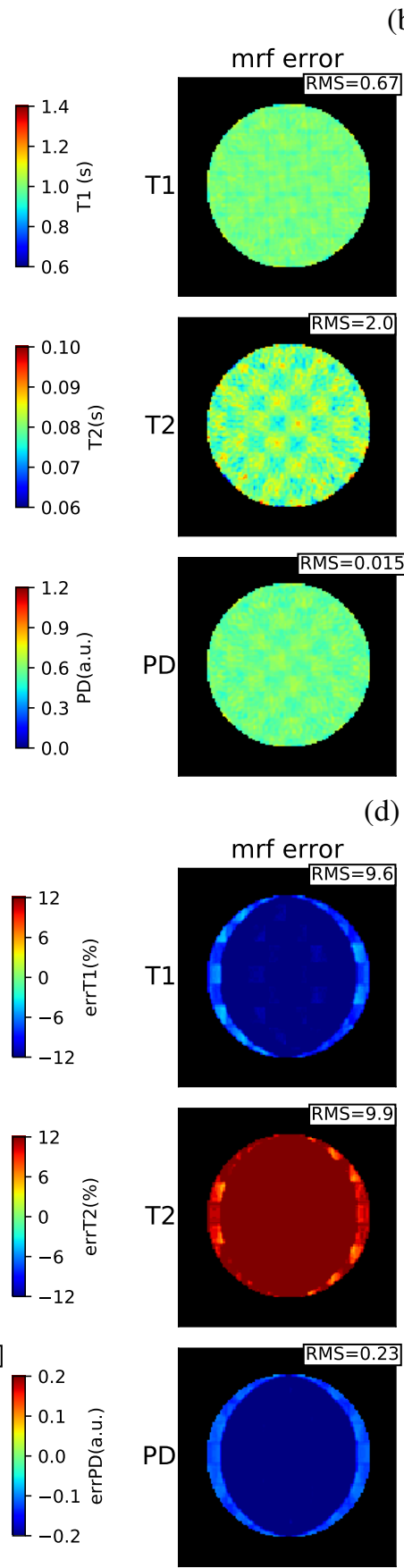

(b) radial

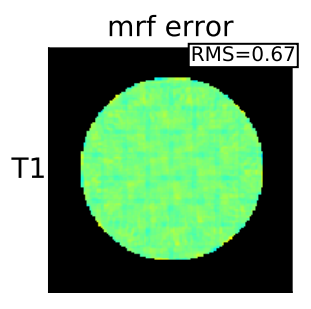

predicted error
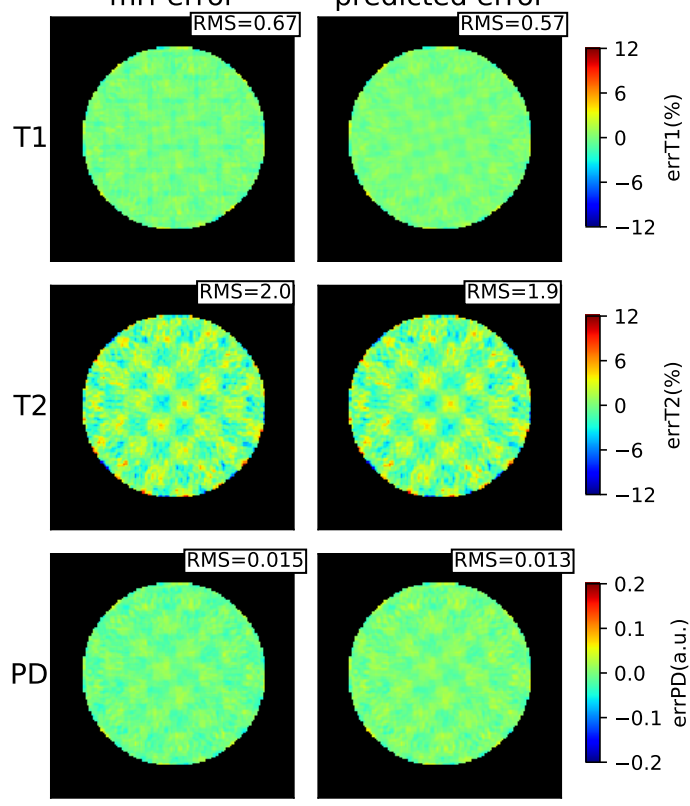

(d) Cartesian
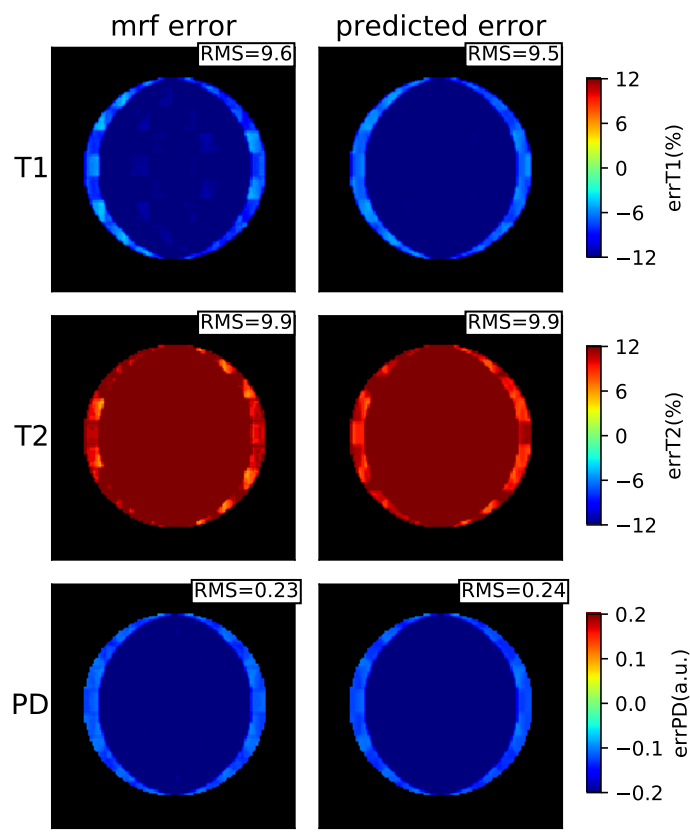

Fig. 2. Test 1.1: A numerical checkerboard phantom. (a) true model and a MRF reconstruction for radial sampling; (b) actual and predicted MRF errors for radial sampling; (c) as (b) for spiral sampling; (d) as (b) for Cartesian sampling. The rows concern $\boldsymbol{T}_{\mathbf{1}}, \boldsymbol{T}_{\mathbf{2}}$ and PD respectively. RMS values are reported in the units of the image they refer to.

TABLE I

PREDicted ERROR VALUes

\begin{tabular}{|c|c|c|c|c|c|c|c|c|c|c|c|c|c|c|c|}
\hline & \multicolumn{15}{|c|}{ Test 1.1 (checkerboard) } \\
\hline & \multicolumn{3}{|c|}{ radial, seq1 } & \multicolumn{3}{|c|}{ spiral, seq1 } & \multicolumn{3}{|c|}{ Cart. seq1 } & \multicolumn{3}{|c|}{ random, seq1 } & \multicolumn{3}{|c|}{ radial, seq2 } \\
\hline & err & $\epsilon_{1}$ & $\epsilon_{2}$ & err & $\epsilon_{1}$ & $\epsilon_{2}$ & err & $\epsilon_{1}$ & $\epsilon_{2}$ & err & $\epsilon_{1}$ & $\epsilon_{2}$ & err & $\epsilon_{1}$ & $\epsilon_{2}$ \\
\hline $\mathrm{T} 1(\%)$ & 0.6 & 0.4 & 0.4 & 2.1 & 2.0 & 0.6 & 12 & 12 & 0.5 & 4.0 & 1.1 & 3.9 & 5.7 & 4.7 & 3.2 \\
\hline $\mathrm{T} 2(\%)$ & 1.9 & 1.1 & 1.6 & 4.6 & 4.4 & 1.3 & 21 & 21 & 0.9 & 7.3 & 4.2 & 5.9 & 6.9 & 5.1 & 4.4 \\
\hline PD $\times 100$ (a.u.) & 1.3 & 0.8 & 1.0 & 4.2 & 4.2 & 0.5 & 24 & 24 & 0.7 & 3.2 & 2.2 & 2.4 & 5.6 & 5.0 & 2.5 \\
\hline & \multicolumn{15}{|c|}{ Test 1.2 (brain phantom) } \\
\hline T1 (\%) & 3.2 & 0.6 & 3.1 & 4.5 & 2.3 & 3.3 & 14 & 13 & 4.5 & 11 & 1.6 & 10 & 30 & 7.4 & 25 \\
\hline $\mathrm{T} 2(\%)$ & 6.0 & 1.1 & 5.9 & 7.7 & 4.6 & 5.1 & 22 & 20 & 4.4 & 22 & 5.2 & 21 & 28 & 7.6 & 25 \\
\hline PD $\times 100$ (a.u.) & 4.1 & 0.8 & 4.1 & 5.9 & 3.5 & 3.8 & 19 & 18 & 4.5 & 9.8 & 2.3 & 9.3 & 22 & 5.8 & 20 \\
\hline
\end{tabular}

The values in the table indicate the RMS of the total error and the partial contributions $\epsilon_{1}$ and $\epsilon_{2}$.

For readibility, the RMSE of the proton density is multiplied by 100 . 
(a) radial
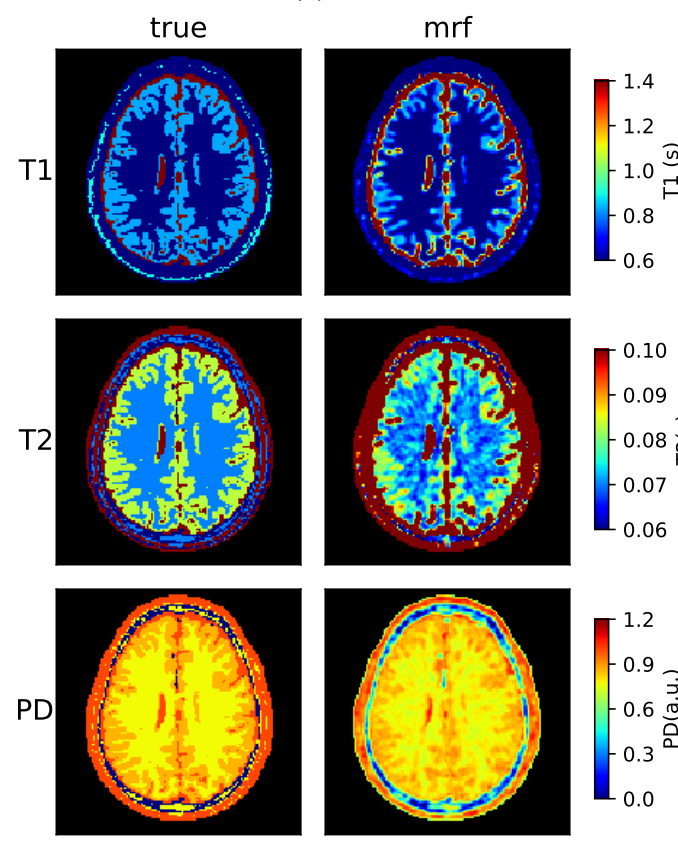

(c) spiral
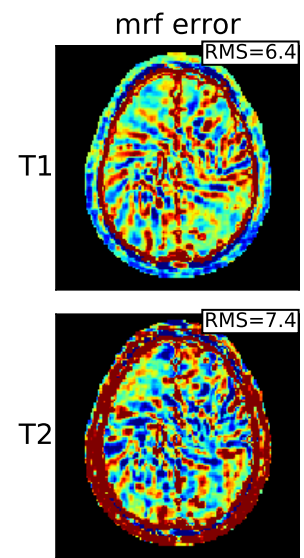

predicted error
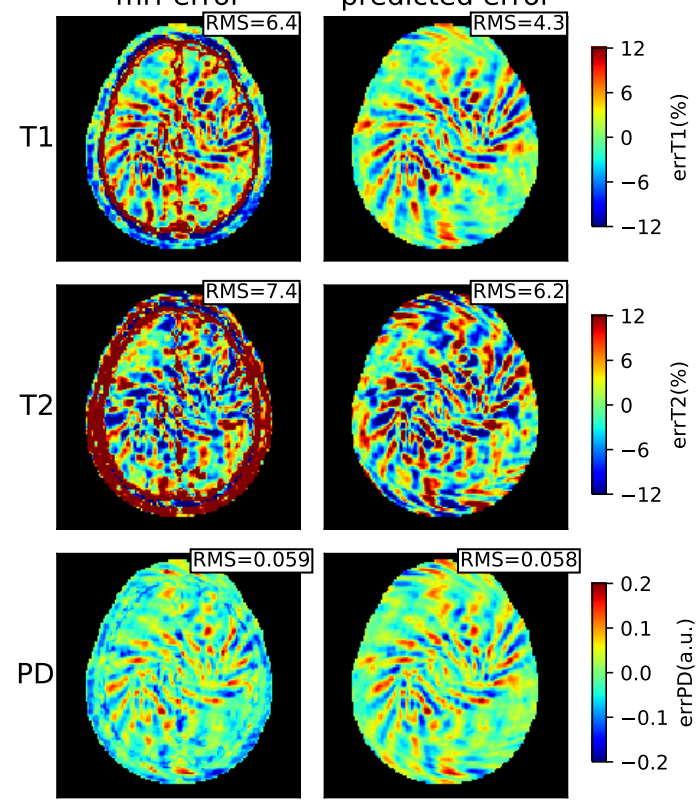

(b) radial

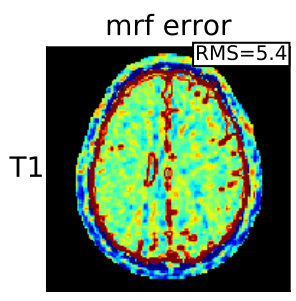

predicted error
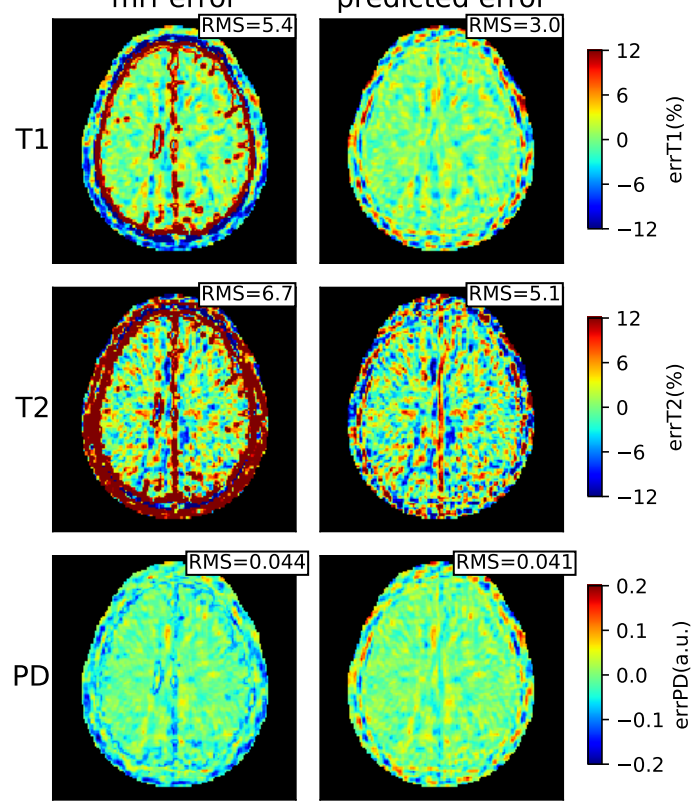

(d) Cartesian
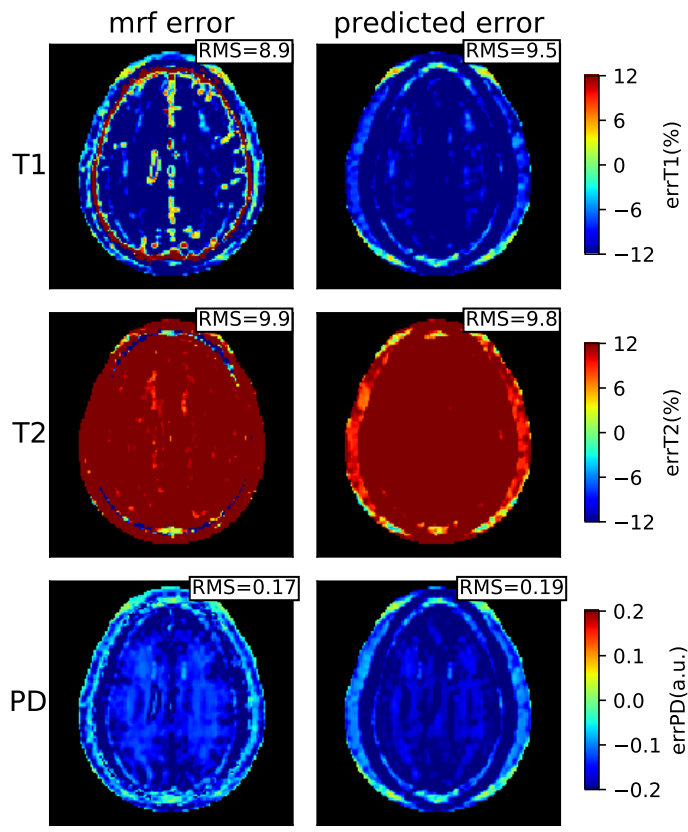

Fig. 3. Test 1.2: A brain phantom. True model, MRF reconstruction, predicted and observed errors as in Figure 2 RMS values are reported in the units of the image they refer to.

In general, $\mathcal{E}_{2}(k)$ strongly depends on $k$, and its values for specific $k$ provide only limited information. Therefore we included plots of the absolute value of $\mathcal{E}_{2}(k)_{p, q}$ as a function of $k$ for $N_{\mathrm{US}}=32$, see Figure 4 In addition, the second line of Table II contains RMS values of the errors $\mathcal{E}_{2}(k)_{p, q}$ for different values of $N_{\text {US }}$. All these data show that the offdiagonal $(2,1)$ components are relatively large. This indicates that the reconstructed $T_{2}$ maps will be strongly affected by the actual $T_{1}$ maps.

In conclusion, we have already noted (see Table \) that RMS values are in general larger for $T_{2}$ maps. In this section we have shown that this is partially due to cross-talk effects, which can be severe especially for the transverse relaxation rate.

\section{The role of randomness and the type of $R F$ excitation.}

Since its conception, randomness has been a fundamental component of the MRF framework. The general understanding is that randomness in $k$-space sampling and/or $\mathrm{RF}$ excitation trains promotes richness of encoding and better reconstructions. To test this assumption, a similar analysis as in the previous subsection is performed for a radial $k$-space sampling scheme in which the angles are randomly permuted. 
TABLE II

CROSS-TALK ERROR MATRIX $\mathcal{E}_{2}(k)$

\begin{tabular}{|l|ccccc|}
\hline & $N_{\text {US }}=8$ & \multicolumn{2}{|c}{16} & 32 & 64 \\
\hline Example: $k=(0.08,0.08) \pi$, errors & $\left(\begin{array}{ll}0.00 & 0.01 \\
0.03 & 0.01\end{array}\right)$ & $\left(\begin{array}{cc}-0.03 & 0.06 \\
0.17 & -0.04\end{array}\right)$ & $\left(\begin{array}{cc}-0.03 & 0.05 \\
0.17 & -0.04\end{array}\right)$ & $\left(\begin{array}{cc}-0.01 & 0.07 \\
0.18 & -0.02\end{array}\right)$ \\
\hline Golden angle k-space sampling & $\left(\begin{array}{ll}0.00 & 0.03 \\
0.05 & 0.03\end{array}\right)$ & $\left(\begin{array}{ll}0.02 & 0.05 \\
0.12 & 0.07\end{array}\right)$ & $\left(\begin{array}{ll}0.02 & 0.05 \\
0.12 & 0.07\end{array}\right)$ & $\left(\begin{array}{ll}0.07 & 0.07 \\
0.13 & 0.17\end{array}\right)$ \\
$|k|=0.25 \pi$, RMS errors & $\left(\begin{array}{ll}0.13 & 0.05 \\
0.22 & 0.16\end{array}\right)$ & $\left(\begin{array}{ll}0.21 & 0.07 \\
0.35 & 0.22\end{array}\right)$ & $\left(\begin{array}{ll}0.30 & 0.10 \\
0.56 & 0.32\end{array}\right)$ & $\left(\begin{array}{cc}0.43 & 0.15 \\
0.76 & 0.46\end{array}\right)$ \\
\hline Random k-space sampling & $k \mid=0.25 \pi$, RMS errors &
\end{tabular}

Fourier analysis of contrast dependent errors

$(p, q)=(1,1)$

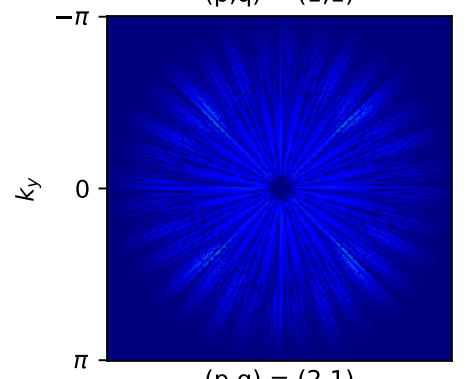

$(p, q)=(2,1)$

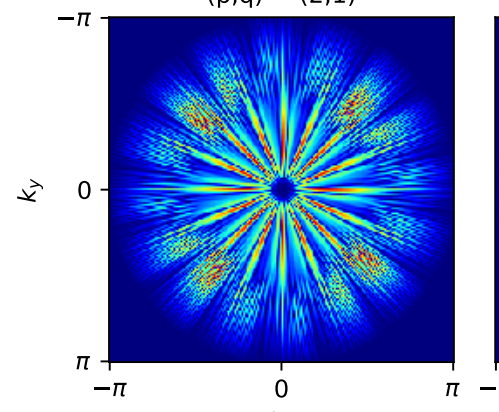

$k_{x}$

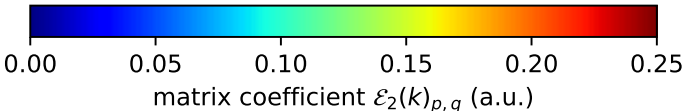

matrix coefficient $\mathcal{E}_{2}(k)_{p, q}$ (a.u.)

Fig. 4. Fourier analysis of contrast dependent errors: Plots of $\mathcal{E}_{2}(k)_{p, q}$ as a function of $k$ for $p, q=1,2$. Note the relatively large coefficients for $(p, q)=(2,1)$. These lead to parameter cross-talk, mapping $T_{1}$ contrast in the true object to $\boldsymbol{T}_{2}$ contrast in the reconstructions.

RMS averaged values of $\mathcal{E}_{2}(k)$ (over $k$ values and random realizations) are given in the third row of Table III and are much larger than the previous, golden angle scheme. We therefore expect the performance of MRF reconstruction to be considerably worse for this randomized acquisition scenario. Our prediction is confirmed by the RMS error values obtained from this scheme, which are included in Table I Clearly, random $k$-space sampling does not necessarily lead to good MRF imaging.

To understand the reason for this, we investigate the dependence of the $\widehat{S}^{(\alpha, \beta)}(k)$ on the sampling scheme. In analogy to Eq. 30p, let's consider the factor $\sum_{r}(\operatorname{Re} N)_{p, r}^{-1} \widehat{S}_{r, q}^{(1,1)}(k)$ for some fixed $k$, and write

$$
\sum_{r}(\operatorname{Re} N)_{p, r}^{-1} \widehat{S}_{r, q}^{(1,1)}(k)=\left\langle f, g_{p, q}\right\rangle
$$

where, to simplify the notation, we define $f, g \in \mathbb{C}^{N_{\mathrm{I}}}$ having components $f(j)=\widehat{P}_{j}(k)$ and $g_{p, q}(j)=$

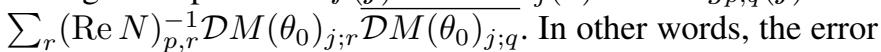
terms are decomposed into a $k$-space sampling dependent part $(f)$ and an RF pulse train dependent part $\left(g_{p, q}\right)$. Let $\widetilde{f}(\nu)$ denote the Fourier transform of $f$, given by $\widetilde{f}(\nu)=$ $\sum_{j=1}^{N_{\mathrm{I}}} f(j) e^{-2 \pi i \nu(j-1)}, \nu=0, \ldots, N_{\mathrm{I}}-1$, and similar for $\widetilde{g}_{p, q}$. From elementary Fourier theory it follows that

$$
\sum_{r}(\operatorname{Re} N)_{p, r}^{-1} \widehat{S}_{r, q}^{(1,1)}(k)=\frac{1}{N_{\mathrm{I}}}\left\langle\widetilde{f}, \widetilde{g}_{p, q}\right\rangle .
$$

In addition, from Eq. (6) and Eq. 19] we have that

$$
\sum_{r}(\operatorname{Re} N)_{p, r}^{-1} \widehat{S}_{\text {mean } ; r, q}^{(1,1)}(k)=\frac{1}{N_{\mathrm{I}}} \tilde{f}(0) \overline{\widetilde{g}_{p, q}(0)} .
$$

Therefore $\sum_{r}(\operatorname{Re} N)_{p, r}^{-1} \widehat{S}_{\text {resid } ;, q, q}^{(1,1)}(k)$ is given by the sum in Eq. (32) where $\nu=0$ is omitted:

$$
\sum_{r}(\operatorname{Re} N)_{p, r}^{-1} \widehat{S}_{\mathrm{resid} ; r, q}^{(1,1)}(k)=\frac{1}{N_{\mathrm{I}}} \sum_{\nu=1}^{N_{\mathrm{I}}-1} \tilde{f}(\nu) \overline{\widetilde{g}_{p, q}(\nu)} .
$$

An inspection of these Fourier transform terms (See Supplementary material section 5) reveals the following behavior for $\widetilde{f}$ and the $\widetilde{g}_{p, q}$ in the case of radial sampling and RF pulse sequence 1 . A large part of the energy of the $\widetilde{g}_{p, q}$ (the $\mathrm{RF}$ excitation dependent terms) is contained in the diagonal coefficients (i.e. matrix indices $p=q$ ) with $\nu=0$, while the higher Fourier coefficients decay rapidly as a consequence of the smoothness of the magnetization response (Fig. S2(a)). At the same time, the energy in $\widetilde{f}$ (the $k$-space sampling dependent term) is concentrated in a few, regularly spaced peaks as a consequence of the highly structured, golden angle $k$-space sampling scheme. The distance between these peaks is such that $\widetilde{g}$ is already negligible at the peak locations with $\nu \neq$ 0 (Fig. S3(a)). Therefore, the sum (34) and the corresponding error term are relatively small. Suppose now that the $k$-space sampling scheme is replaced by its randomized version. In this case, larger values of $\widetilde{f}$ at low but nonzero $\nu$ lead to larger values of the sum in Eq. (34) (Fig. S3(b)). This explains the larger errors for the random sampling.

Let us now consider the RF dependent term, $g$, for a different flip angle train. To this aim, we introduce a new sequence, called sequence 2, which is displayed in Fig. 5 The RMS error values obtained for this sequence are given in the right section of Table I According to these results, the non-smooth Sequence 2 leads to larger MRF reconstruction errors in the tissue parameters. This fact is easily explained at the hand of Eq. (34). Non-smooth magnetization responses have slower decay of Fourier coefficients. This will naturally 

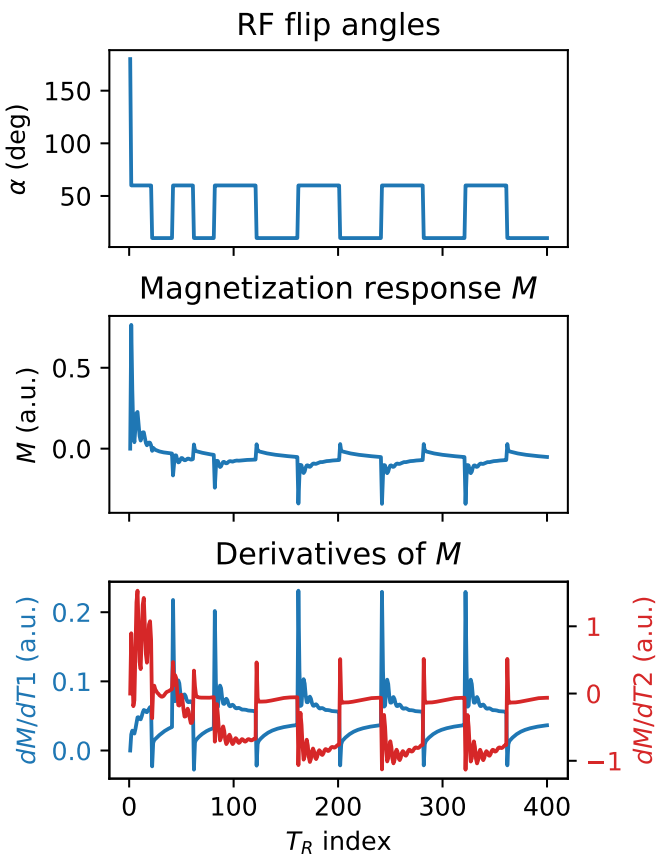

Fig. 5. Flip angles and plot of $M(t, \theta)$ and some derivatives for $\boldsymbol{T}_{\mathbf{1}}=$ $1.0 \mathrm{~s}, \boldsymbol{T}_{2}=0.08 \mathrm{~s}, \mathrm{PD}=1$ (a.u.), for sequence 2 .

lead to larger values of Eq. (34), which is exactly what we observe for sequence 2 (Fig. S2(b)).

In conclusion, Eq. (34) suggests that the RF pulse train and the $k$-space sampling scheme should destructively interfere (or, in mathematical terms, be orthogonal) in the Fourier domain. This fact is reminiscent of the incoherence between encoding and sparsity transforms in the compressed sensing framework [19]. Temporal randomness in either $k$-space or RF excitation is just a particular way to achieve incoherence in MRF. In fact, as it has been shown in recent work [9], [15], [16], randomly perturbed sequences do not have additional value with respect to encoding performance and smooth RF trains can indeed perform very well. The analysis from this section provides an explanation for this fact and a criterion for optimizing the acquisition protocol.

\section{DISCUSSION}

We have provided a mathematical analysis of the combined effects of $k$-space sampling and RF transient state excitation in the error of MRF reconstructions. The perturbations can be decomposed, to a first order approximation, into two main terms $\left(\epsilon_{1}\right.$ and $\left.\epsilon_{2}\right)$ whose structure and behavior have been analyzed. Numerical simulations for different acquisition strategies from Section [V] show that our model is accurate and can predict the actual reconstruction error even for realistic anatomies (brain). An in-depth analysis performed in Section $\nabla$ revealed that inter-parameter cross-talk can be a substantial issue, especially for the $T_{2}$ values. Furthermore, randomness in $k$-space and/or RF excitation train appears to play a secondary role or to be even sub-optimal. As a culmination of our analysis, we illustrated how the RF and $k$-space sampling interact and jointly contribute to the reconstruction error. An incoherence criterion for improved MRF protocol is outlined which ensures destructive interference of the corresponding two terms in the Fourier domain.

In section III a model for the MRF estimate $\theta^{*}$ was obtained by expressing the parameters $\theta$ as a first order expansion $\theta(x)=\theta_{0}+\theta_{1}(x)$ where $\theta_{0}$ is treated as a constant or as a binary mask. We have shown that when $\theta_{0}$ is close to $\theta$, our model performs very well. For brain imaging, $T_{1}$ and $T_{2}$ values of white and gray matter are in fact relatively close to the average of the two which can be used as reference value. Note that gray and white matter are critical tissues for brain imaging. On the other hand, cerebrospinal fluid (CSF) has relatively much larger $T_{1}$ and $T_{2}$ values but we have shown that the proposed approach is still able to model the error in a satisfactory way.

The primary reason for the derivation of our error model is to provide insights in the working of MRF. Compared to direct voxel-by-voxel dictionary match, our model makes it possible to analyze and reveal important MRF features in a generalized context. In particular, the use of the functions (convolution kernels) $\widehat{S}_{1}^{(\alpha, \beta)}(k)$ allows to draw conclusions valid, simultaneously, for all object parameters and all kind of sequence choices such as RF train, $k$-space coverage and under-sampling strategies. For example, in section $\mathrm{V}$, we have formally derived the beneficial effect of frequent sampling in the center of $k$-space. This result might not surprise the reader since it is somehow intuitively understood. On the other hand, we have shown why Cartesian sampling is to be avoided in MRF, a fact which explains the few applications of Cartesian schemes in this paradigm.

Probably more interesting are the results obtained regarding the role of randomness and irregularity in the sequence design. We have shown that a well designed sequence does not necessarily need to include randomization and in fact this feature could degrade the performance of the method; the $\mathrm{RF}$ train envelope and $k$-space sampling scheme should be mutually destructively interfering in the Fourier domain. One straightforward way to achieve this is to employ a regular golden angle radial (or spiral) trajectory with a smooth RF excitation train. However, this is only a possibility and more efficient combinations could be found. Further investigation into this direction would go beyond the scope of this paper and is left to future studies.

Our error model, in combination with a signal term and possibly other hardware constraints, can be leveraged also for algorithmic optimization of the sequence; parameters such as the number of acquisition intervals (snapshots), the values of the RF flip angles, the type of $k$-space sampling scheme, the echo-time and repetition time, whether to use gradient spoiling or not, jointly and directly influence our error model and thus can be effectively optimized at once. We believe that this is what distinguishes our approach to previous sequence design work where either the $k$-space sampling is not taken into account or it is handled separately from the RF pulse design. In addition, we stress the fact that a pre-computed dictionary is not needed since the dependency of the error on the sequence parameters can be quantified purely at the hand of our model. This is a fundamental advantage for iterative sequence optimization which otherwise would require the 
construction of a dictionary for each new choice of sequence parameters.

As the method is based on first order Taylor expansion, questions regarding the effects of higher order terms might arise. However, while it is possible to include some higher order terms, it is not clear that this will lead to substantially better error estimates, since the convergence of the perturbation expansion is not guaranteed.

In this study we have focused on the mathematical analysis of the MRF framework. Since a ground truth is required for error quantification, the validation and interpretation of our model were carried out at the hand of numerical simulations on realistic models and scenarios. We believe the results from the numerical tests provide sufficient explanation and illustration of the theoretical findings. Therefore, acquired in-vivo data from MRI systems was not taken into consideration.

A reader familiar with the field of inversion theory might expect such an approach to the analysis of MRF reconstructions. Hoewever, although there are some developments towards the application of inverse theory in multi-parametric quantitative MRI (see [4], [7], [9], [15], [20]), the dictionarymatch approach is still the most adopted. In other words, we are interested in the mainstream implementation of MRF, which, from a mathematical perspective, is probably more challenging than the inverse problem theory. While extensive theoretical results are available for inversion problems in general (and with this we include the parametric reconstruction for Gaussian distributed noise), there is very limited work which addresses the sensitivity of the dictionary match to the case of non-Gaussian artifacts. With this work, we aim at filling this gap. An analysis of MRF for an inversion approach would result into a rather different methodology and thus it would go beyond the scope of this paper.

The popularity of MRF is mainly a consequence of its good empirical performance. We hope that this work will inspire researchers in the field to apply our analysis to other scenarios. In particular, several extensions to this work can be investigated which could not find place in our study. For instance, the signal model can be modified to include diffusion effects, transmit RF system inhomogeneity, slice profile response [21], balanced gradient trajectories. A thorough understanding of MRF from a theoretical point of view is necessary to pave the way for its application in the clinical setting. This work could represent a step in this direction.

\section{REFERENCES}

[1] Dan Ma, Vikas Gulani, Nicole Seiberlich, Kecheng Liu, Jeffrey L Sunshine, Jeffrey L Duerk, and Mark A Griswold. Magnetic resonance fingerprinting. Nature, 495(7440):187-192, 2013.

[2] Yun Jiang, Dan Ma, Nicole Seiberlich, Vikas Gulani, and Mark A Griswold. MR fingerprinting using fast imaging with steady state precession (FISP) with spiral readout. Magnetic resonance in medicine, 74(6):1621-1631, 2015.

[3] Martijn A Cloos, Florian Knoll, Tiejun Zhao, Kai T Block, Mary Bruno, Graham C Wiggins, and Daniel K Sodickson. Multiparametric imaging with heterogeneous radiofrequency fields. Nature Communications, 7:12445, 2016.

[4] Mike Davies, Gilles Puy, Pierre Vandergheynst, and Yves Wiaux. A compressed sensing framework for magnetic resonance fingerprinting. SIAM Journal on Imaging Sciences, 7(4):2623-2656, 2014.
[5] Debra F McGivney, Eric Pierre, Dan Ma, Yun Jiang, Haris Saybasili, Vikas Gulani, and Mark A Griswold. SVD compression for magnetic resonance fingerprinting in the time domain. IEEE Transactions on Medical Imaging, 33(12):2311-2322, 2014.

[6] Mariya Doneva, Thomas Amthor, Peter Koken, Karsten Sommer, and Peter Börnert. Matrix completion-based reconstruction for undersampled magnetic resonance fingerprinting data. Magnetic resonance imaging, 41:41-52, 2017.

[7] Jakob Assländer, Martijn A Cloos, Florian Knoll, Daniel K Sodickson, Jürgen Hennig, and Riccardo Lattanzi. Low rank alternating direction method of multipliers reconstruction for MR fingerprinting. Magnetic Resonance in Medicine, 79(1):83-96, 2018.

[8] Bo Zhao, Kawin Setsompop, Huihui Ye, Stephen F Cauley, and Lawrence L Wald. Maximum likelihood reconstruction for magnetic resonance fingerprinting. IEEE Transactions on Medical Imaging, 35(8):1812-1823, 2016

[9] Alessandro Sbrizzi, Tom Bruijnen, Oscar van der Heide, Peter Luijten, and Cornelis AT van den Berg. Dictionary-free MR Fingerprinting reconstruction of balanced-GRE sequences. arXiv preprint arXiv:1711.08905, 2017.

[10] Leslie Greengard and June-Yub Lee. Accelerating the nonuniform fast Fourier transform. SIAM review, 46(3):443-454, 2004.

[11] Jeffrey A. Fessler and Bradley P. Sutton. Nonuniform fast Fourier transforms using min-max interpolation. IEEE Transactions on Signal Processing, 51(2):560-574, 2003.

[12] Stephen F Cauley, Kawin Setsompop, Dan Ma, Yun Jiang, Huihui Ye, Elfar Adalsteinsson, Mark A Griswold, and Lawrence L Wald. Fast group matching for MR fingerprinting reconstruction. Magnetic Resonance in Medicine, 74(2):523-528, 2015.

[13] Yong Chen, Yun Jiang, Shivani Pahwa, Dan Ma, Lan Lu, Michael D Twieg, Katherine L Wright, Nicole Seiberlich, Mark A Griswold, and Vikas Gulani. MR fingerprinting for rapid quantitative abdominal imaging. Radiology, 279(1):278-286, 2016.

[14] Jesse I Hamilton, Yun Jiang, Yong Chen, Dan Ma, Wei-Ching Lo, Mark Griswold, and Nicole Seiberlich. MR fingerprinting for rapid quantification of myocardial T1, T2, and proton spin density. Magnetic Resonance in Medicine, 77(4):1446-1458, 2017.

[15] Bo Zhao, Justin P Haldar, Congyu Liao, Dan Ma, Mark A Griswold, Kawin Setsompop, and Lawrence L Wald. Optimal experiment design for magnetic resonance fingerprinting: Cramer-rao bound meets spin dynamics. arXiv preprint arXiv:1710.08062, 2017.

[16] Jakob Assländer, Riccardo Lattanzi, Daniel K Sodickson, and Martijn A Cloos. Relaxation in spherical coordinates: Analysis and optimization of pseudo-SSFP based MR-Fingerprinting. arXiv preprint arXiv:1703.00481, 2017.

[17] Jeff Bezanson, Alan Edelman, Stefan Karpinski, and Viral B Shah. Julia: A fresh approach to numerical computing. SIAM Review, 59(1):65-98, 2017.

[18] RK-S Kwan, Alan C Evans, and G Bruce Pike. MRI simulationbased evaluation of image-processing and classification methods. IEEE Transactions on Medical Imaging, 18(11):1085-1097, 1999.

[19] Michael Lustig, David. L1 Donoho, Juan M. Santos, and John M. Pauly. Compressed sensing MRI. IEEE Signal Processing Magazine, 25(2):7282, 2008.

[20] Alessandro Sbrizzi, Oscar van der Heide, Martijn Cloos, Annette van der Toorn, Hans Hoogduin, Peter R. Luijten, and Cornelis A.T. van den Berg. Fast quantitative MRI as a nonlinear tomography problem. Magnetic Resonance Imaging, 46:56-63, 2018.

[21] Dan Ma, Simone Coppo, Yong Chen, Debra F McGivney, Yun Jiang, Shivani Pahwa, Vikas Gulani, and Mark A Griswold. Slice profile and B1 corrections in 2D magnetic resonance fingerprinting. Magnetic Resonance in Medicine, 78(5):1781-1789, 2017. 


\title{
Understanding the combined effect of $k$-space undersampling and transient states excitation in MR Fingerprinting reconstructions. Supplementary Material
}

\author{
Christiaan C. Stolk and Alessandro Sbrizzi
}

\section{Perturbation theory with variable reference proton density}

We proceed with the derivation of the equivalent of Eq. (22) from the main text in the case of spatiallydependent reference proton density, $\rho_{0}(x)$. We still assume there is a constant reference value $\eta_{0}$ for the other parameters. Recall from section II the notation $\theta=(\eta, \rho)$ or $\theta=(\eta, \operatorname{Re} \rho, \operatorname{Im} \rho)$.

In case of a real proton density, we express the quantitative parameters $\theta(x)$ in terms of the spatially dependent $\rho_{0}(x)$, the constant $\theta_{0}$ and spatially dependent contrast functions $\eta_{1}(x)$ and $\rho_{1}(x)$ as follows

$$
\theta(x)=\left(\eta_{0}+\eta_{1}(x), \quad \rho_{0}(x)\left(1+\rho_{1}(x)\right)\right) .
$$

Similarly, in the case of a complex proton density, we write

$$
\theta(x)=\left(\eta_{0}+\eta_{1}(x), \quad \operatorname{Re}\left(\rho_{0}(x)\left(1+\rho_{1}(x)\right)\right), \quad \operatorname{Im}\left(\rho_{0}(x)\left(1+\rho_{1}(x)\right)\right)\right) .
$$

This choice allows us to exploit the linearity of the signal with respect to the proton density. In particular, the decomposition $\rho(x)=\rho_{0}(x)\left(1+\rho_{1}(x)\right)$ makes it possible to bring the spatial dependent reference $\rho_{0}(x)$ outside the signal equation and treat the remaining part $1+\rho_{1}(x)$ as a constant plus a spatial dependent contrast terms, which is the same scenario considered in Section III. Therefore, we wil be able to use the same definitions for $S_{p, q}^{(1,1)}(x)$ etc. from subsection III-B of the main text.

We can apply the perturbation theoretic analysis of Section III with constant reference $\theta_{0}$ and spatial dependent contrast term $\theta_{1}(x)$ defined in the following way. In case of real $\rho$ we write

$$
\begin{aligned}
\theta_{0} & =\left(\eta_{0}, 1\right) \\
\theta_{1}(x) & =\left(\eta_{1}(x), \rho_{1}(x)\right) .
\end{aligned}
$$

For complex $\rho$ we write

$$
\begin{aligned}
\theta_{0} & =\left(\eta_{0}, 1,0\right) \\
\theta_{1}(x) & =\left(\eta_{1}(x), \operatorname{Re} \rho_{1}(x), \operatorname{Im} \rho_{1}(x)\right) .
\end{aligned}
$$

With these definitions, Eq. (2) from Section II is modified to

$$
s_{j, l}=\sum_{x \in G_{\mathrm{p}}} \rho_{0}(x) M_{j}\left(\theta_{0}+\theta_{1}(x)\right) e^{i k_{j, l} \cdot x} .
$$

Note that only the multiplicative factor $\rho_{0}$ has been added to the original signal equation. This is a consequence of our choice for the definition of $\theta$ in Eq. (2) above.

In the absence of thermal noise, the undersampled images are given by

$$
\tilde{I}_{j}(x)=P_{j} *\left(\rho_{0}(\cdot) M_{j}\left(\theta_{0}+\theta_{1}(\cdot)\right)\right)(x) .
$$


For the estimates $\theta^{*}(x)$ we use similar redefinitions, writing $\theta^{*}$ in terms of a variable reference proton density

$$
\theta^{*}(x)=\left(\eta_{0}^{*}+\eta_{1}^{*}(x), \rho_{0}^{*}(x)\left(1+\rho_{1}^{*}(x)\right)\right)
$$

By inserting the definition for $\theta$ and $\theta^{*}$ the stationarity equations become

$$
\begin{aligned}
0= & \operatorname{Re} \sum_{j=1}^{N_{\mathrm{I}}}\left|\rho_{0}^{*}(x)\right|^{2} \overline{D M\left(\theta_{0}+\theta_{1}^{*}(x)\right)_{j ; p}} M\left(\theta_{0}+\theta_{1}^{*}(x)\right)_{j} \\
& -\operatorname{Re} \sum_{j=1}^{N_{\mathrm{I}}} \sum_{y \in G_{\mathrm{p}}} P_{j}(x-y) \overline{\rho_{0}^{*}(x)} \overline{D M\left(\theta_{0}+\theta_{1}^{*}(x)\right)_{j ; p}} \rho_{0}(y) M\left(\theta_{0}+\theta_{1}(y)\right)_{j} .
\end{aligned}
$$

In the above equation, the factor $\overline{\rho_{0}^{*}}$ can be taken out of the sum. The resulting equation can be expanded in a similar way as Eq. (15) of the main text. The formal first order expansion is

$$
\begin{array}{r}
0=\operatorname{Re}\left(\overline { \rho _ { 0 } ^ { * } } \left[\rho_{0}^{*} S_{0 ; p}^{(1,0)} * 1(x)+\sum_{q} \rho_{0}^{*} \theta_{1, q}^{*} S_{0 ; p, q}^{(1,1)} * 1(x)+\sum_{q} \rho_{0}^{*} \theta_{1, q}^{*} S_{0 ; p, q}^{(2,0)} * 1(x)\right.\right. \\
-S_{0 ; p}^{(1,0)} * \rho_{0}(x)-\sum_{q} S_{0 ; p, q}^{(1,1)} *\left(\rho_{0} \theta_{1}\right)(x)-\sum_{q} \theta_{1, q}^{*} S_{0 ; p, q}^{(2,0)} * \rho_{0}(x) \\
\left.\left.-S_{1 ; p}^{(1,0)} * \rho_{0}(x)-\sum_{q} S_{1 ; p, q}^{(1,1)} *\left(\rho_{0} \theta_{1}\right)(x)-\sum_{q} \theta_{1, q}^{*} S_{1 ; p, q}^{(2,0)} * \rho_{0}(x)\right]\right) .
\end{array}
$$

In this equation, we can still choose the function $\rho_{0}^{*}(x)$. In order to obtain some cancellations, we set

$$
\rho_{0}^{*}(x)=P * \rho_{0}(x)
$$

Looking carefully at the definitions of $S_{0 ; p}^{(1,0)}$ and of $S_{0 ; p, q}^{(2,0)}$ it follows that the first and fourth terms in (9) cancel each other. The same holds for the third and sixth term. The remaining equation is

$$
\begin{array}{r}
0=\operatorname{Re}\left(\overline { \rho _ { 0 } ^ { * } ( x ) } \left[\sum_{q} \rho_{0}^{*} \theta_{1, q}^{*} S_{0 ; p, q}^{(1,1)} * 1(x)-\sum_{q} S_{0 ; p, q}^{(1,1)} *\left(\rho_{0} \theta_{1}\right)(x)\right.\right. \\
\left.\left.-S_{1 ; p}^{(1,0)} * \rho_{0}(x)-\sum_{q} S_{1 ; p, q}^{(1,1)} *\left(\rho_{0} \theta_{1}\right)(x)-\sum_{q} \theta_{1, q}^{*} S_{1 ; p, q}^{(2,0)} * \rho_{0}(x)\right]\right) .
\end{array}
$$

This is the perturbation theoretic expansion of the stationarity equations in case of a variable reference density $\rho_{0}(x)$. Equation (27) in the main text follows from Eq. (11) in an analogous way as Eq. (25) follows from Eq. (22).

\section{Simulation details}

Signal simulations are based on the Bloch equations in rotating coordinates. RF pulses are modeled using an instantaneous rotation with a given flip angle and phase, while between RF pulses and readout times the usual exponential decays are applied.

To model dephasing due to gradient spoiling, each voxel is assumed to consist of $N_{\mathrm{GS}}$ isochromats. Each isochromat is rotated by a different phase factor. The phase factors were distributed uniformly over the interval $[0,2 \pi]$. Here $N_{\mathrm{GS}}=128$ was chosen.

Non-uniform Fourier transform reconstructions are performed by the NUFFT package of reference [1]. The fundamental issue in the image reconstruction is the choice of the $k$-space weights $w_{j, l}$. These should be set such that the resulting point spread function is a bandlimited Dirac delta function that is smoothly 
(a)

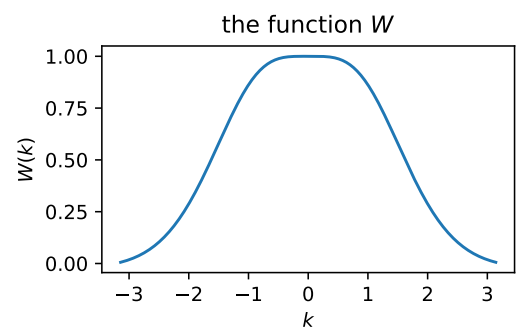

Figure 1: The smooth, low pass $k$-space windowing function employed for the reconstruction of the snapshots images.

truncated in the Fourier domain, as a hard truncation leads to oscillatory nonlocal contributions (Gibbs phenomenon). In radial sampling, we aim at

$$
\widehat{P}(k)=W(|k|),
$$

where $W$ is a smooth window function on $[-\pi, \pi]$, see Fig. 1. To obtain this, the density of $k$-space sampling must be taken into account. Relative to Nyquist sampling, this density, averaged over circles of constant $|k|$, is given by

$$
\begin{aligned}
\text { relativeDensity }(|k|) & =\text { relativeAngularDensity } \cdot \text { relativeRadialDensity } \\
& =\frac{2}{|k|} \frac{1}{N_{\mathrm{US}}} \cdot \text { relativeRadialDensity } .
\end{aligned}
$$

Based on this, the weights $w_{j, l}$ were defined by

$$
w_{j, l}= \begin{cases}W\left(\left|k_{j, l}\right|\right) / \text { relativeDensity }\left(\left|k_{j, l}\right|\right) & \text { if } k \neq 0 \\ C_{\mathrm{R}, 0} & \text { if } k=0\end{cases}
$$

where $C_{\mathrm{R}, 0}$ is such that the Fourier transform of the PSF at $k=0$ (approximated by applying the PSF to the function 1 ) is equal to 1 at $x=0$. For spirally sampled $k$-space the weights were defined by

$$
w_{j, l}=W\left(\left|k_{j, l}\right|\right) / \text { relativeDensitySpiral }\left(\left|k_{j, l}\right|\right),
$$

resulting in a similar point spread function. For Cartesian sampling the weights were given by

$$
w_{j, l}=W\left(\left|\left(k_{j, l}\right)_{1}\right|\right) W\left(\left|\left(k_{j, l}\right)_{2}\right|\right) N_{\mathrm{US}}
$$

In this case the point spread function was similarly well-localized as the radial and spiral ones.

\section{Further examples for validation}

The checkerboard example in section IV-B of the main text has a constant proton density and moderate variations in $T_{2}$. For the purpose of validation two more checkerboard examples with larger variations in these parameters are shown in Figure 2. 
(a) radial
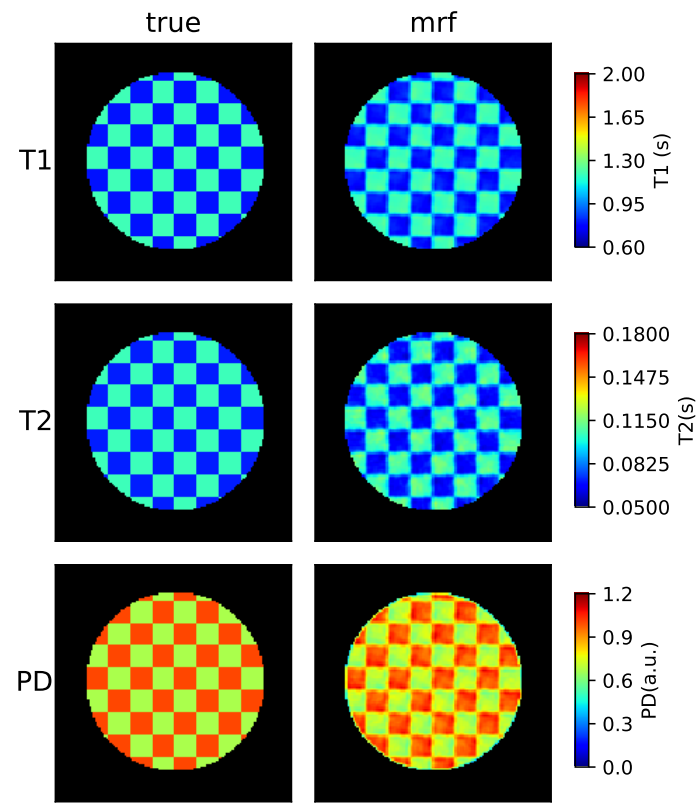

(c) spiral
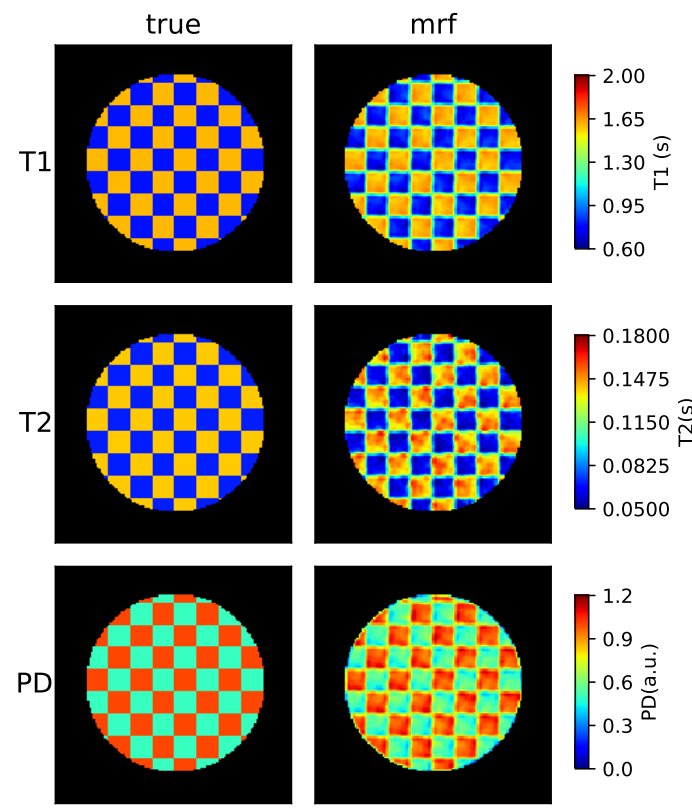

(b) radial
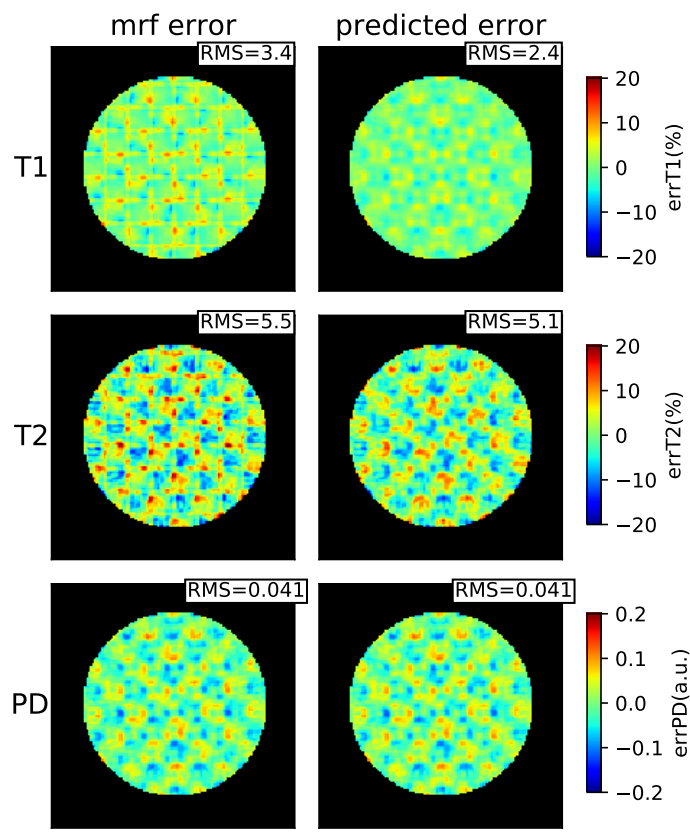

(d) Cartesian
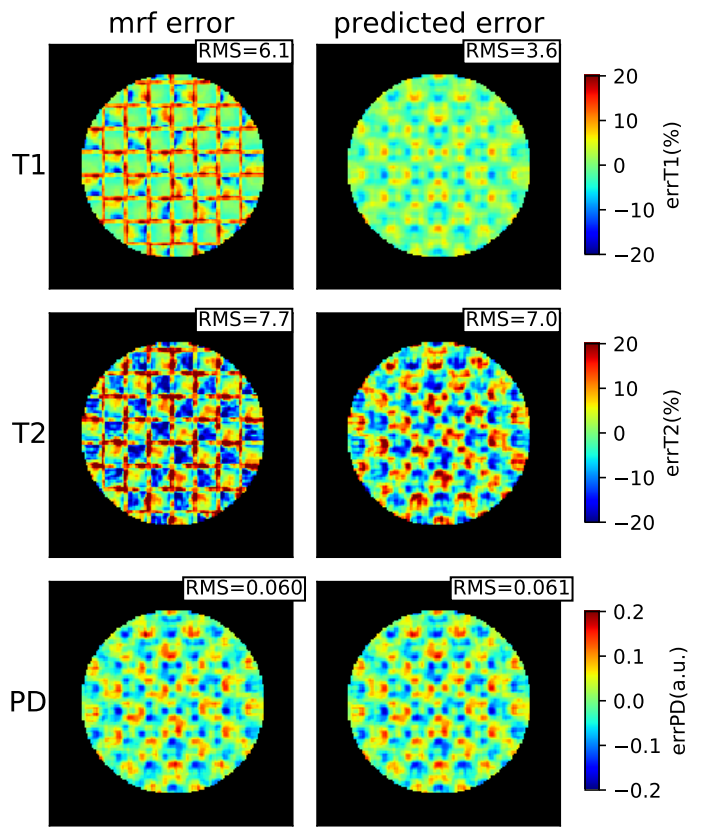

Figure 2: Numerical checkerboard phantoms with contrast factors 1.5 and 2. (a,c) true model and MRF reconstructions; (b,d) actual and predicted MRF errors for radial sampling. Parameters values in (a) $T_{1}$ : $(0.8,1.2) \mathrm{s}, T_{2}:(0.07,0.105) \mathrm{s}, \mathrm{PD}:(0.67,1.0)$ a.u. Parameter values in $(\mathrm{c}): T_{1}:(0.8,1.6) \mathrm{s}, T_{2}:(0.07$, $0.14) \mathrm{s}, \mathrm{PD}:(0.5,1.0)$ a.u. 


\section{Analysis of the undersampling errors $e_{\mathrm{US}}$}

In Figure 3 some data is shown that confirm that, in general, the undersampling errors $e_{\mathrm{US}}$ are not independent and identically distributed Gaussian noise.

(a)
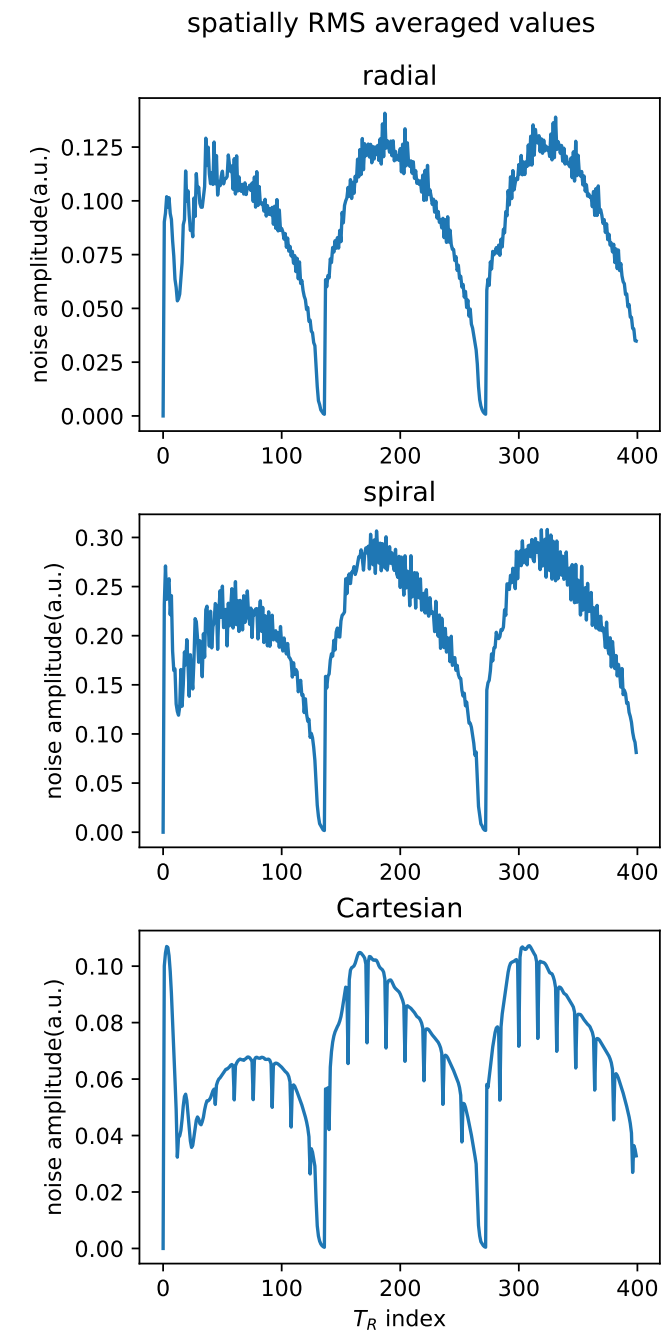

Figure 3: Spatial RMS averages of the undersampling errors $e_{\mathrm{US}, j}$ as a function of time for the sequence described in section IV-A applied to the brain phantom described in IV-C. 


\section{$5 \quad$ Figures for section V-C}
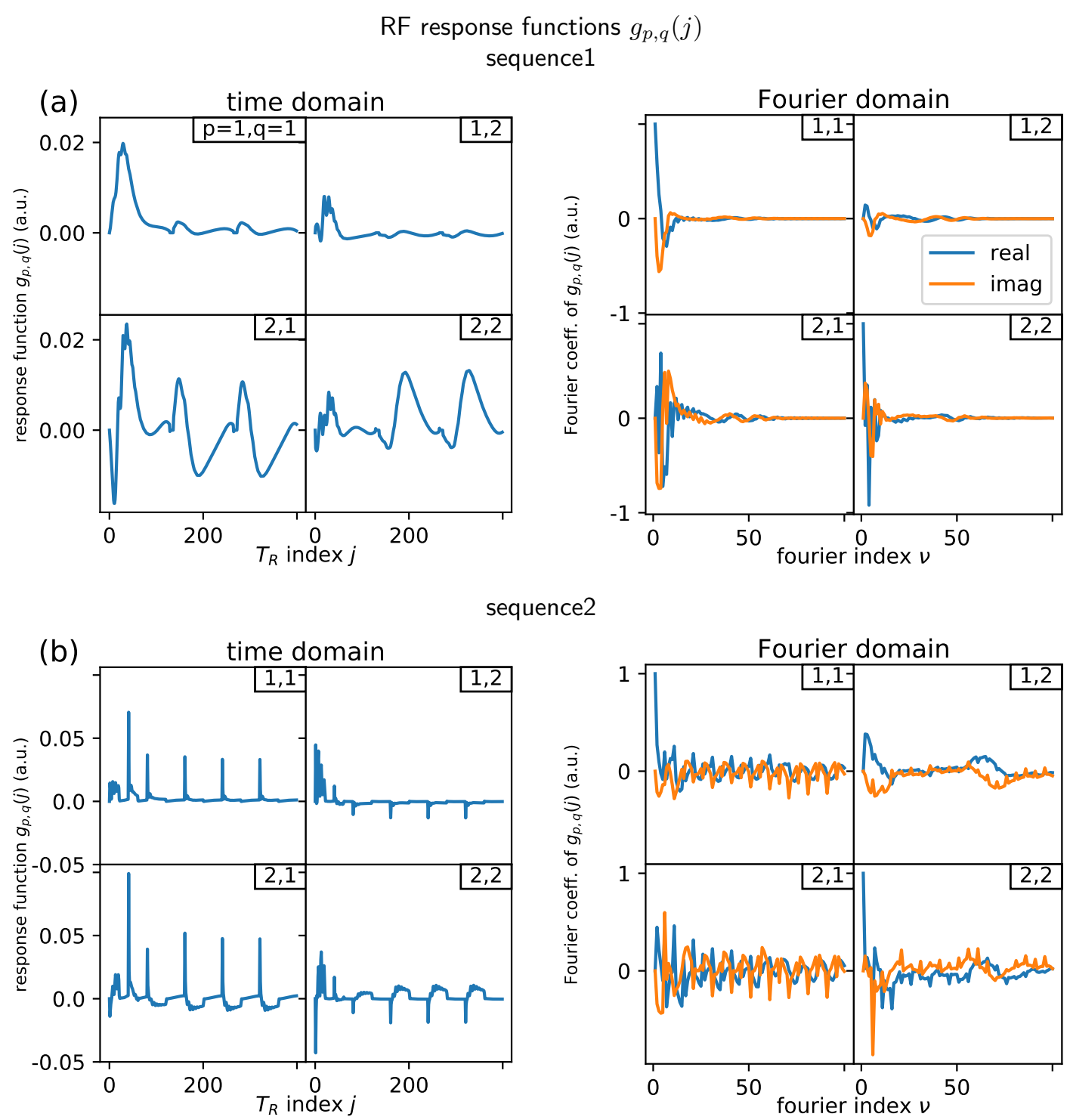

Figure 4: The time-dependent matrix components $g_{p, q}(j)$ and their Fourier transforms for (a) sequence 1 and (b) sequence 2. See section V-C. Note the rapid decay of the Fourier coefficients in sequence 1. For sequence 2, the decay is much slower and the coefficients are large also for $\nu \gg 0$. Note also that for sequence 1 , the $(2,1)$ coefficient appears to be relatively large compared to the other matrix components. This fact can explain the substantial crosstalk in the reconstructed $T_{2}$ (see also section V-B). 

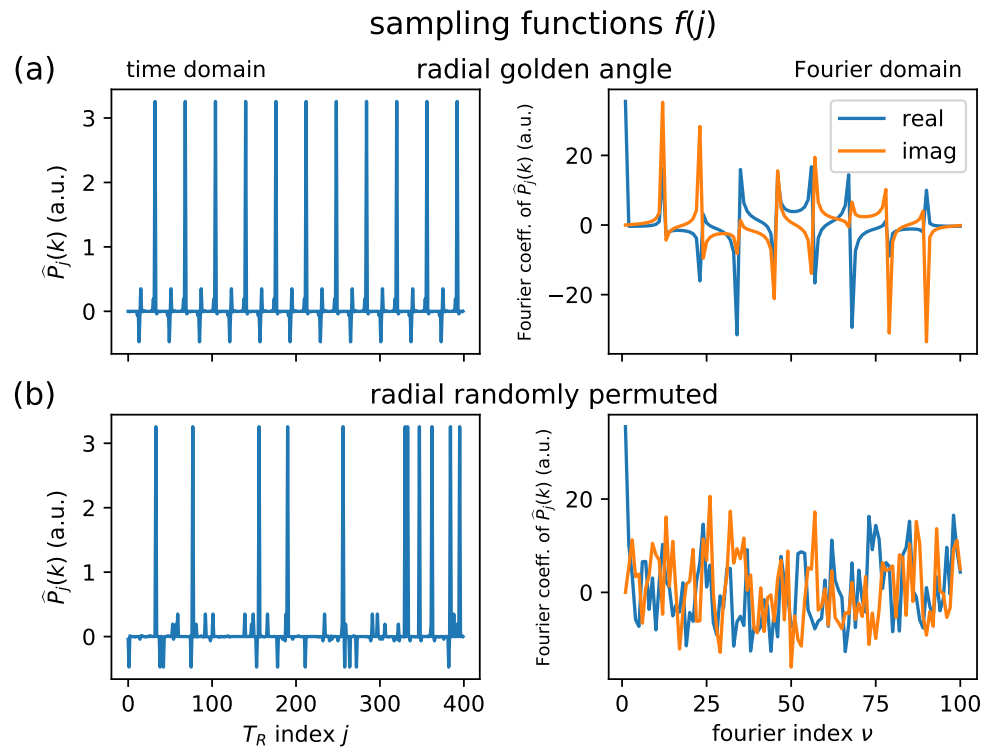

Figure 5: Plots of the time-dependent sampling schemes $f_{j}=\widehat{P}_{j}(k), j=1, \ldots, N_{\mathrm{I}}$ and their Fourier transform in $j$. See section V-C. Two sampling schemes are considered: (a) golden angle radial sampling and (b) randomly permuted radial sampling. The undersampling factor is 32 and a random value of $k$ with $|k|=0.25 \pi$ is chosen. The behavior is independent of the specific choice of $k$. For the golden angle sampling, the regular pattern in the time domain leads to regularly spaced peaks in the Fourier domain. For randomly permuted radial sampling, such regularly spaced peaks are absent and the Fourier transform exhibits a typical random behavior.

\section{References}

[1] Leslie Greengard and June-Yub Lee. Accelerating the nonuniform fast Fourier transform. SIAM review, 46(3):443-454, 2004. 